\title{
Wall-modeled large-eddy simulation of the flow past a rod-airfoil tandem by the Lattice Boltzmann method
}

\author{
H. Touil ${ }^{(1)}$, S. Malik ${ }^{(2)}$, E. Lévêque ${ }^{(2)}$, D. Ricot ${ }^{(3)}$ and A. Sengissen ${ }^{(4)}$ \\ (1) C-S Systèmes d'Information, Lyon, France \\ (2) LMFA, CNRS, Ecole Centrale de Lyon, Ecully, France \\ (3) Technocentre Renault, Guyancourt, France \\ (4) Airbus Operations SAS, Toulouse, France
}

\begin{abstract}
Purpose - The lattice Boltzmann (LB) method offers an alternative to conventional computational fluid dynamics (CFD) methods. However, its practical use for complex turbulent flows of engineering interest is still at an early stage. In this article, a LB wallmodeled large-eddy simulation (WMLES) solver is outlined. The flow past a rod-airfoil tandem in the sub-critical turbulent regime is examined as a challenging benchmark.

Design/methodology/approach - Fluid dynamics are discretized upon the LB principles. The large-eddy simulation is accounted straightforwardly by including a modeled subgrid-scale viscosity in the LB scheme, whereas a wall-law model enforces the boundary condition at the first off-wall node. This physical modeling is briefly introduced and relevant references are given for details. The flow past a rod-airfoil tandem at Reynolds number $\operatorname{Re}=4.8 \times 10^{4}$ and Mach number $\mathrm{Ma} \simeq 0.2$ is simulated on a composite multiresolution grid; the numerical set-up is detailed. Unsteady aerodynamic and aeroacoustic features including spectral analysis and far-field pressure fluctuations are discussed.

Findings - Extensive quantitative comparisons with both experimental and numerical reference data indicate that aerodynamic and aeroacoustic features are well captured by the LB simulation.
\end{abstract}

Originality/value - Our study shows that WMLES within the LB framework provides a workable and efficient alternative to Navier-Stokes CFD solvers in the context of complex turbulent flows. The LB method permits to access an attractive turnaround time while 
preserving engineering accuracy.

Keywords: Aerodynamics, Aeroacoustics, Rod-airfoil benchmark, Lattice Boltzmann method, Wall-modeled large-eddy simulation.

Paper type: Research paper

\section{Nomenclature}

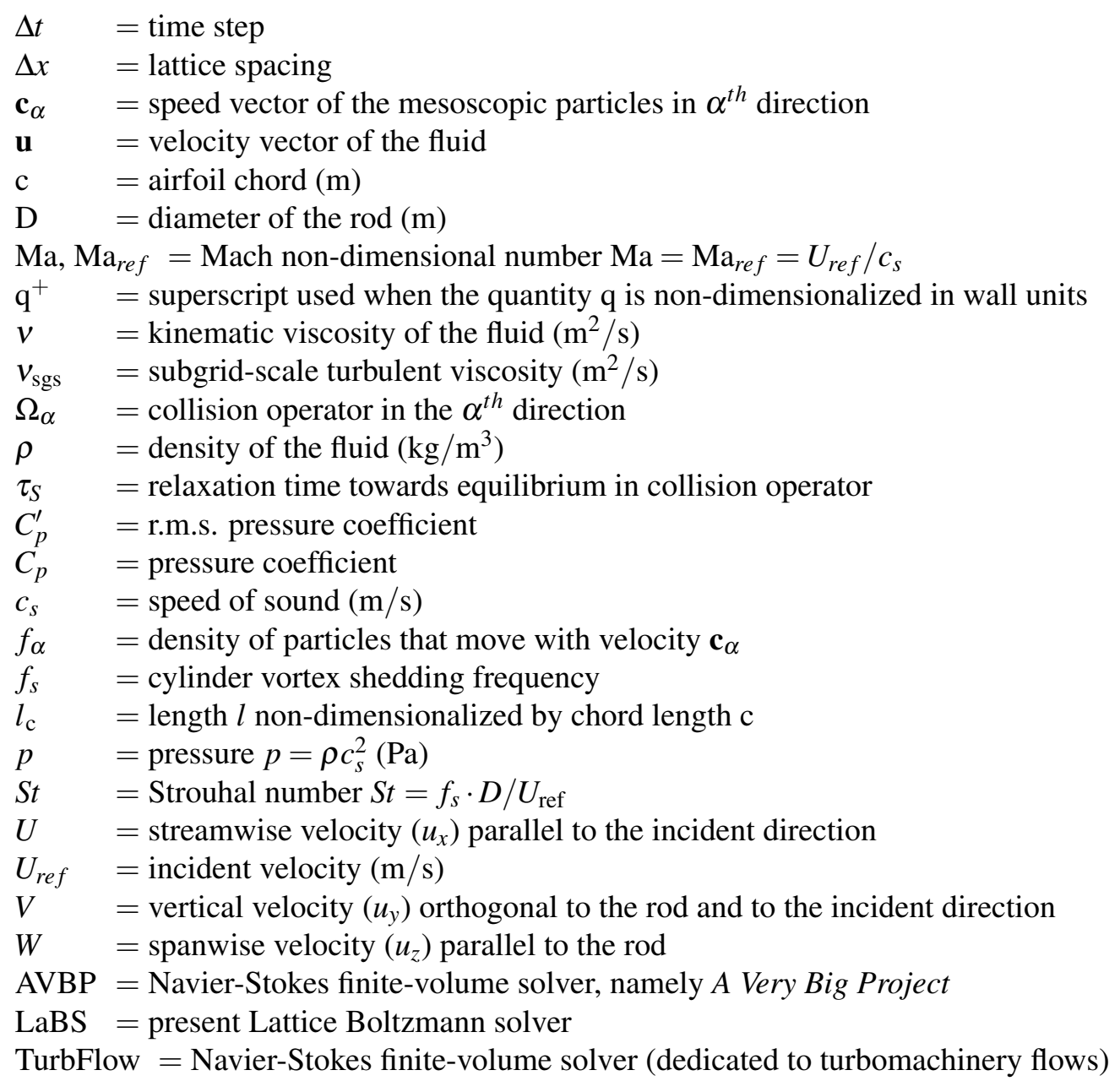




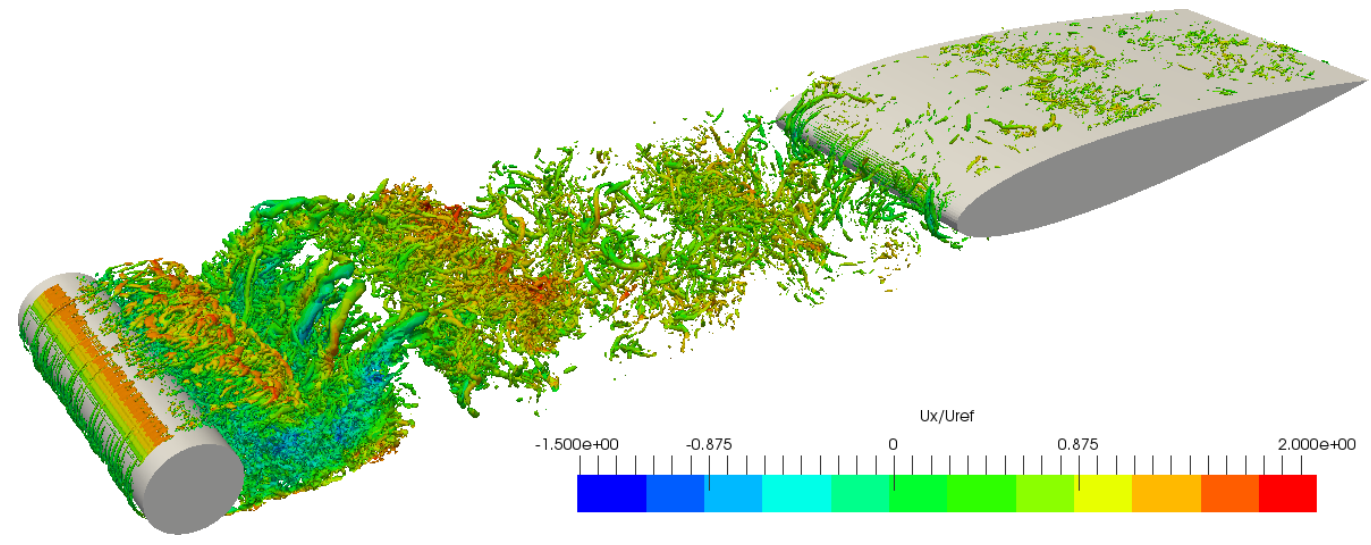

Figure 1: Wall-modeled LES of the flow past a rod-airfoil tandem at $\operatorname{Re}_{\mathrm{D}}=4.8 \times$ $10^{4}$ in the subcritical turbulent regime (shear-layer transition regime) by the lattice Boltzmann method. Instantaneous iso-surfaces of Q-criterion colored by the nondimensional streamwise velocity $U / U_{\text {ref. }}$.

\section{Context and motivations}

Standard approaches of computational fluid dynamics (CFD) rely on the discretization of the Navier-Stokes (N-S) equations, which govern dynamics at a macroscopic level. The N-S equations are physically sound, however, their numerical integration is difficult owing mainly to their highly non-linear nature. For most cases of interest, solutions develop many scales of motion and levels of amplitude that are difficult to handle numerically, and require to resort to high-order discretization methods and specific meshing strategies [42]. These constraints are strengthened when addressing complex turbulent flows.

In the last three decades, the lattice Boltzmann (LB) method has emerged as a conceptually different approach of CFD. The first computationally viable realization dates back to the late eighties [38]. The LB scheme governs fluid motions at a mesoscopic level that is intermediate between the microscopic and the macroscopic [10, 37]. Capturing the kinetic behavior of collections of fluid particles is here preferred to solving non-linear PDEs. This seems to be a crazy bet, however, most details at the mesoscopic level actually play no role at the macroscopic level. Therefore, much simplier mesoscopic dynamics may be designed retaining only the basic features that pertain at the macroscopic level. This is, in short, the rationale behind the LB approach [24, 17]. Considerable success in simulating fluid flows and heat transfer problems have already been reported [2, 39] but its 
practical use for turbulent flows of engineering interest remains at an early stage as compared to classical NS approaches [9, 32]. Our study helps to fill this gap by assessing quantitatively the potential of the LB approach in terms of accuracy, numerical workability and turnaround time on a complex flow configuration. The flow past a rod-airfoil tandem in the sub-critical turbulent regime is considered as a challenging test case. Our study complements some preliminary LB results already reported on this test case [34]. In such flow configuration, the LB approach must incorporate turbulence modeling and deploy on a composite multi-resolution grid to focus on large-eddy dynamics and alleviate computational efforts [40]. For this purpose, a modeled subgrid-scale viscosity is included in the bulk dynamics, whereas a wall model is used to enforce a boundary condition at the first off-wall node without resolving the whole boundary layer. This eventually yields a intricate set of advanced numerical methods and physical models bundled in a CFD solver that is here tested.

The rod-airfoil flow configuration is recognized as a representative benchmark for the numerical modeling of turbulent fluid motions interacting with airframe elements [18]. It is recommended by the Advisory Group for Aerospace Research and Development (AGARD). The rod creates a turbulent wake which interacts with the airfoil downstream. At diameter-based Reynolds number $\operatorname{Re}_{\mathrm{D}}=$ $4.8 \times 10^{4}$, the boundary layer separation on the rod occurs upstream the transition. This results in a three-dimensional turbulent vortex shedding that eventually impinges onto the airfoil and partly splits at its leading edge (see Fig. 1). The rodairfoil flow in the shear-layer transition regime (or sub-critical turbulent regime) is well-documented. It involves not only complex dynamics related to turbulent von Kármán street and wake impingement, but also non-trivial sound generation processes [19]. This test-case has been considered to evaluate the capability of our lattice Boltzmann wall-modeled large-eddy simulation (WMLES) solver. Importantly, detailed quantitative comparisons have also been made with conventional high-order finite-volume N-S solvers on the same flow configuration and comparable numerical set-up for a better evaluation.

The numerical method and the physical modeling into play are presented in section 2 with a particular emphasis on the LB scheme and the turbulence modeling, which are original. The numerical set-up of the simulation is detailed in section 3 while the results are discussed in section 4 . Concluding remarks are given at the end. 


\section{Numerical method and physical modeling}

\subsection{Lattice Boltzmann method}

Our solver is built upon the LB principles, which offers a particle-based description of fluid dynamics $[10,37]$. Therefore, the fluid is viewed as populations of fictitious particles (carrying mass) that collide and move along the links of a discrete Cartesian lattice. This obviously refers to a kinetic description and rigorous connections can be established with the Boltzmann equation [36].

Usual macroscopic fluid motions are reconstructed locally by summing up the contributions of particles moving in the different directions. The mass density $\rho$ and fluid momentum $\rho \mathbf{u}$ are given at each lattice node by

$$
\rho=\sum_{\alpha} f_{\alpha} \quad \text { and } \quad \rho \mathbf{u}=\sum_{\alpha} f_{\alpha} \mathbf{c}_{\alpha}
$$

where $f_{\alpha}$ denotes the density of particles that move with a discrete velocity $c_{\alpha}$. The D3Q19 lattice with nineteen possible velocities is here adopted. It is sketched in Fig. 2.

The LB scheme governs the evolution in space and time of the $f_{\alpha}$ 's on the lattice and usually proceeds in a two-step collide-and-stream procedure $[10,37]$. The collision accounts for the instantaneous redistribution of particles among the different directions of propagation and expresses formally as

$$
f_{\alpha}^{\text {out }}(\mathbf{x}, t)=f_{\alpha}(\mathbf{x}, t)+\Omega_{\alpha}(\mathbf{x}, t),
$$

where $\Omega_{\alpha}$ can be identified as a collision operator. The streaming transports the particles (according to their post-collision velocity) to the neighboring lattice nodes, i.e.

$$
f_{\alpha}\left(\mathbf{x}+\mathbf{c}_{\alpha} \Delta t, t+\Delta t\right)=f_{\alpha}^{\text {out }}(\mathbf{x}, t) .
$$

These two steps eventually yield the fundamental equation of the LB scheme

$$
f_{\alpha}\left(\mathbf{x}+\mathbf{c}_{\alpha} \Delta t, t+\Delta t\right)=f_{\alpha}(\mathbf{x}, t)+\Omega_{\alpha}(\mathbf{x}, t) .
$$

This equation is reminiscent of the discrete-velocity Boltzmann equation integrated along characteristics.

The fluid mechanics is introduced essentially through the modeling of the collision operator. In this respect, the so-called BGK approximation, initially proposed by Bhatnagar Gross and Krook [3] is often used:

$$
\Omega_{\alpha}(\mathbf{x}, t)=-\frac{1}{\tau_{S}}\left(f_{\alpha}(\mathbf{x}, t)-f_{\alpha}^{\mathrm{eq}}(\mathbf{x}, t)\right) .
$$




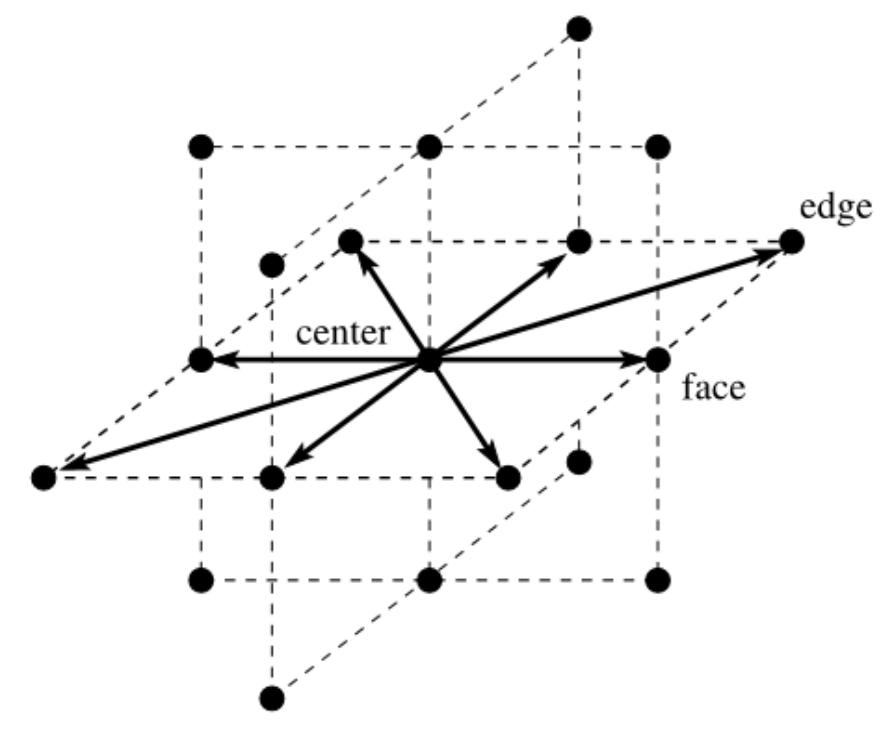

Figure 2: The set of particle velocities $\left\{\mathbf{c}_{\alpha}\right\}$ at each node of the D3Q19 lattice. These 19 velocities can be grouped into three categories of vectors pointing respectively to the "center" (null velocity), the "faces" and the "edges" of a cube. For clarity, only the velocities in the horizontal plane are displayed. During a time step, the particles move exactly from a node to a neighbouring node of the lattice.

This approximation refers to the relaxation of all densities to their values at (isothermal) statistical equilibrium

$$
f_{\alpha}^{\mathrm{eq}}=w_{\alpha} \rho\left(1+\frac{u_{i} c_{\alpha i}}{c_{s}^{2}}+\frac{u_{i} u_{j} Q_{\alpha_{i j}}}{2 c_{s}^{4}}\right) \quad \text { with } \quad Q_{\alpha i j}=c_{\alpha_{i}} c_{\alpha_{j}}-c_{s}^{2} \delta_{i j}
$$

Repeated indices $i, j$ are implicitly summed over in Eq. (6). The weighting coefficients are given by $w_{0}=1 / 3$ for the "center", $w_{1 \ldots 6}=1 / 18$ for the "faces" and $w_{7 \ldots 18}=1 / 36$ for the "edges". To ensure physical consistency, $c_{s}$ refers to the speed of sound in the fluid and the relaxation coefficient $\tau_{S}$ is linked to the kinematic shear viscosity of the fluid by $\tau_{S}=1 / 2+v / c_{s}^{2} \Delta t$. In practice, dynamics is expressed in lattice units for which the time step and the lattice spacing are both equal to unity. Furthermore, $\Delta x / \Delta t=\sqrt{3} c_{s}$ by construction. This scheme is explicit, second-order accurate in time and in lattice spacing and approaches (at low frequency) the solution of the weakly compressible isothermal Navier-Stokes equations with a third-order error in Mach number. Finally, the implicit equation 
of state for the fluid is $p=\rho c_{s}^{2}$.
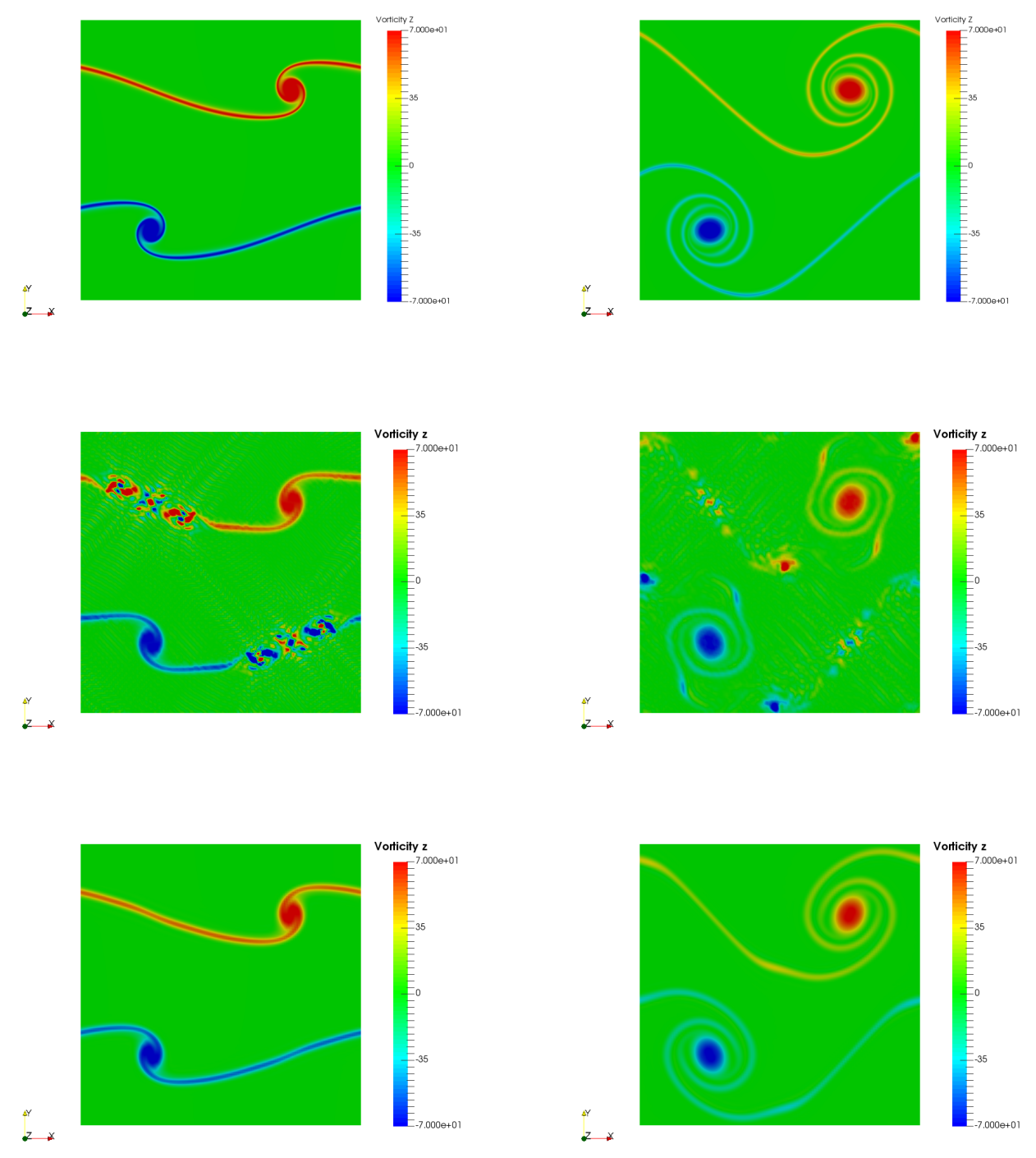

Figure 3: Doubly periodic shear layers vorticity z-component distribution at times $t=0.6 \mathrm{~s}$ (left) and $t=1.0 \mathrm{~s}$ (right). From top to bottom: BGK reference solution on a $1024^{2}$ grid, BGK on a $128^{2}$ grid and DRT scheme with $\tau_{n}=0.7$ on a $128^{2}$ grid 


\subsection{Lattice Boltzmann solver}

\subsubsection{Collision operator}

Our algorithm is a variant of the standard BGK scheme introduced above. It is based on a two-relaxation-time collision operator supplemented with high-order selective filters applied to flow variables for a better robustness and accuracy [30]. Its abbreviated name is DRT for dual relaxation time in the following. The idea behind this variant is to over-relax the distributions towards equilibrium while preserving the conservation of mass and momentum, and to reconstruct the nonequilibrium part of the distributions through a second-order regularization procedure according to [21]. This modified collision expresses as

$$
\begin{gathered}
\widetilde{f}_{\alpha}^{\text {out }}=f_{\alpha}-\frac{1}{\tau_{n}}\left(f_{\alpha}-f_{\alpha}^{\mathrm{eq}}\right) \\
f_{\alpha}^{\text {out }}=\widetilde{f}_{\alpha}^{\text {out }}+\frac{\tau_{S}-\tau_{n}}{\tau_{n} \tau_{S}} \frac{w_{\alpha}}{2 c_{s}^{4}}\left(c_{\alpha_{i}} c_{\alpha j}-c_{s}^{2} \delta_{i j}\right) \Pi_{i j}^{\text {neq }}
\end{gathered}
$$

where $\Pi_{i j}^{\text {neq }}=\sum_{\alpha}\left(f_{\alpha}-f_{\alpha}^{\mathrm{eq}}\right) c_{\alpha_{i}} c_{\alpha j}$ is the non-equilibrium part of the second-order moment, $\tau_{S}$ is the physical shear relaxation time and $\tau_{n}$ is a purely numerical relaxation time aimed at damping non-hydrodynamical ghost modes and improving the stability. Repeated indices $i, j$ are implicitly summed over in Eq. (8).

To illustrate the efficiency of our specific DRT scheme, the doubly periodic shear layers is used as a standard test case. The initial field reads

$$
\left\{\begin{array}{l}
p\left(x, t_{0}\right)=p_{0} \\
\rho\left(x, t_{0}\right)=\rho_{0} \\
u_{1}\left(x, t_{0}\right)=\left\{\begin{array}{l}
\tanh \left(\theta\left(y-\frac{1}{4}\right)\right), \text { for } y \leq \frac{1}{2} \\
\tanh \left(\theta\left(\frac{1}{4}-y\right)\right), \text { for } y>\frac{1}{2}
\end{array}\right. \\
u_{2}\left(x, t_{0}\right)=\delta \sin \left(2 \pi\left(x+\frac{1}{4}\right)\right. \\
u_{3}\left(x, t_{0}\right)=0
\end{array}\right.
$$

where $\theta=120$ and $\delta=0.05$. These values are chosen in order to compare qualitatively to the reference papers $[6,25,12]$. The z-component of the vorticity at times $t=0.6 \mathrm{~s}$ (left) and $t=1.0 \mathrm{~s}$ (right) is displayed in Fig. 3 for a reference BGK simulation on a high-resolution $1024^{2}$ grid (top), a BGK simulation on a much coarser $128^{2}$ grid (middle) and a DRT simulation with $\tau_{n}=0.7$ on the same coarse grid (bottom). While the BGK simulation suffers from numerical instabilities, the DRT scheme (bottom) obviously shows an improved stability and suitably approximates the high-resolution solution (top). 


\subsubsection{Turbulence modeling}

Unsteady simulations of flows at Reynolds numbers of interest for engineering applications require to resort to large-eddy simulation (LES). In order to make the computation tractable, the grid resolution is deliberately reduced. In that case, the original flow equations must be supplemented by a new term accounting for the interaction between unresolved (subgrid) and resolved dynamics. A common thread is to assume that this stress is essentially diffusive, which calls for the modeling of an additional subgrid-scale viscosity. In the context of engineering flows, which may experience strong unsteady events such as boundary-layer separation, vortex shedding or disturbances induced by a moving body, the modeling of the subgrid-scale viscosity is known as a difficult problem [31]. Strong unsteadiness generally occurs at low frequencies compared to the turbulence activity. Furthermore, it is often associated with large amplitudes of the rate of strain. In this respect, a variant of the Smagorinsky model has been used to account explicitly for the low-frequency variations of the rate of strain [22]. Namely, the viscosity of the shear-improved Smagorinsky model (SISM) formulates as

$$
v_{\mathrm{sgs}}(\mathbf{x}, t)=\left(C_{s} \Delta\right)^{2}(|S|(\mathbf{x}, t)-\mathscr{S}(\mathbf{x}, t))
$$

where $C_{S}=0.18$ is the standard Smagorinsky constant, $\Delta$ is the local grid spacing and $|S|$ denotes the norm of the rate-of-strain tensor, $S_{i j}=1 / 2\left(\partial_{j} u_{i}+\right.$ $\left.\partial_{i} u_{j}\right)$. The correcting term $\mathscr{S}$ is the norm of the low-pass filtering (in time) of the rate of strain, as detailed in $[7,5]$. This modeling in conjunction with the LB approach has already proved to be valuable for the simulation of turbulent flows $[40,33]$. Let us emphasize that LES can be handled straightforwardly within the LB framework [9]. The subgrid-scale viscosity appears as an additional spatiotemporal contribution to the relaxation time, i.e.

$$
\tau_{S}(\mathbf{x}, t)=\frac{1}{2}+\frac{v+v_{\mathrm{sgs}}(\mathbf{x}, t)}{c_{s}^{2} \Delta t} .
$$

Beside this (minor) correction, the scheme remains unchanged.

In order to reduce the computational cost, the solver handles multi-domain grid displaying various levels of spatial resolution. The matching of distributions at the interface between domains accounts for the discontinuity of the rate of strain and encompasses effects related to the subgrid-scale dynamics, as already detailed in [40]. Finally, a wall-law model is used to match the flow variables (at the first 
off-wall cell) with the unresolved boundary-layer dynamics [23]. Our wall law includes adverse-pressure-gradient effects and curvature corrections. Formally,

$$
U^{+}\left(y^{+}\right)=\left(\frac{1}{\kappa} \log y^{+}+B\right)+\text { correcting terms. }
$$

All details about the wall-law modeling may be found in $[1,29]$. The reconstruction (at the boundary) of particle densities from flow variables accounts accurately for the shape of the geometry [41].

\section{Numerical set-up of the test case}

Our flow configuration reproduces the experiment carried out by Jacob et al. in the anechoic wind tunnel at Ecole Centrale de Lyon [18]. Namely, a symmetric NACA0012 airfoil of chord $c=0.1 \mathrm{~m}$ is placed one chord-length downstream a rod of diameter $D=0.01 \mathrm{~m}$. The incoming flow is uniform with velocity $U_{\text {ref }}=$ $72 \mathrm{~m} / \mathrm{s}$, temperature $T=293 \mathrm{~K}$ and mass density $\rho=1.204 \mathrm{~kg} / \mathrm{m}^{3}$. The sound speed is $c_{s}=343 \mathrm{~m} / \mathrm{s}$ yielding a reference Mach number $\mathrm{Ma}_{\mathrm{ref}} \approx 0.2$.

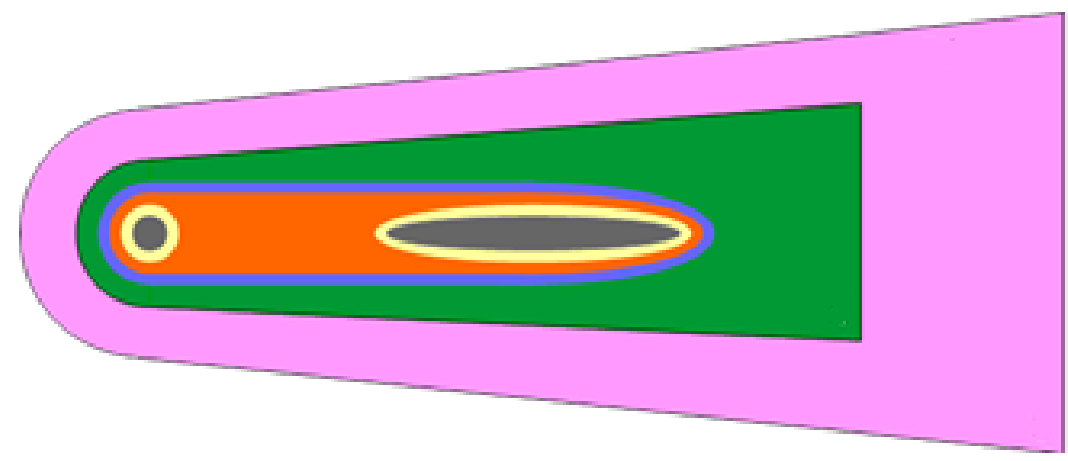

\begin{tabular}{|c|c|c|c|c|c|c|}
\hline$\Delta \mathrm{t}$ & $1.68 \times 10^{-7} \mathrm{~s}$ & $3.36 \times 10^{-7} \mathrm{~s}$ & $6.73 \times 10^{-7} \mathrm{~s}$ & $1.35 \times 10^{-6} \mathrm{~s}$ & $2.69 \times 10^{-6} \mathrm{~s}$ & $5.38 \times 10^{-6} \mathrm{~s}$ \\
\hline$\Delta \mathrm{x}$ & $1 \times 10^{-4} \mathrm{~m}$ & $2 \times 10^{-4}$ & $4 \times 10^{-4} \mathrm{~m}$ & $8 \times 10^{-4} \mathrm{~m}$ & $1.6 \times 10^{-3} \mathrm{~m}$ & $3.2 \times 10^{-3} \mathrm{~m}$ \\
\hline Color & & & & & & \\
\hline
\end{tabular}

Figure 4: The computational mesh is structured in six embedded refinement levels. The external zone (surrounding white zone) is a very large parallelepipedic box, not shown here for clarity. A view of the whole simulation domain is shown in Fig. 10 for instance. 
The computational domain is structured in six embedded zones refined by octree [40] with increasing resolution when approaching the rod and the airfoil (see Fig. 4). The overall number of cubic cells is approximatively $20 \times 10^{6}$. The grid resolution fulfills usual standards for LES [31]. The domain is periodic in the spanwise direction and extends over 0.35 chord length. Friction-less conditions are used for the top and bottom boundaries. Let us mention that the external mesh (white zone) is here not sufficiently broad to allow sound waves to propagate to the far field.

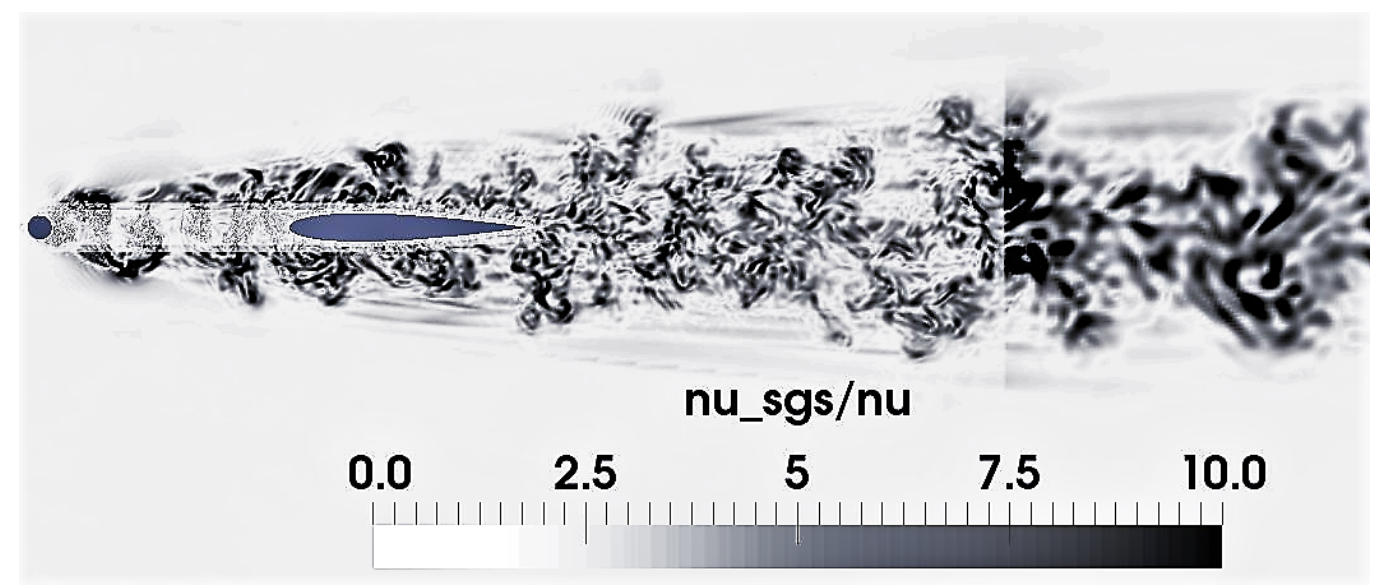

Figure 5: Snapshot of the subgrid-scale activity identified by the ratio $v_{\mathrm{sgs}}(\mathbf{x}, t) / v$ with $v_{\text {sgs }}(\mathbf{x}, t)$ given by Eq. (10).

The grid resolution in the vicinity of the rod and the airfoil is $\Delta x / c=0.001$ corresponding approximatively to $\Delta x^{+} \approx 20$ in wall units. This justifies the introduction of a wall law to treat the boundary condition at the first off-wall cell. In the bulk of the flow, the unresolved dynamics due to the lack of grid resolution is accounted by the subgrid-scale viscosity given by Eq. (10). As mentioned previously, this viscosity is included as an additional contribution to the relaxation time $\tau_{S}$ in the collision process according to Eq. (11). As expected, Fig. 5 shows that the subgrid-scale viscosity prevails over the fluid viscosity in regions where turbulent motions develop. The turbulence model truly takes the control of the dissipation in these regions. Note also that the intensity of the subgrid-scale viscosity increases as the grid becomes coarser. Finally, dynamic sponge zones [11] are used to avoid spurious reflections of acoustic waves on each side of the computational domain except for the periodic direction. The efficiency of these 
sponge zones, where pressure fluctuations are strongly damped, is highlighted in Fig. 10.

For the validation of our simulation, the results are compared with the experimental measurements reported in [18] and alternative wall-resolved NavierStokes LES performed on the same flow configuration with the AVBP (A Very Big Project) and TurbFlow solvers. Let us mention that the mesh resolution is globally higher in the two wall-resolved N-S simulations than in our wall-modeled LB simulation. The AVBP solves the compressible N-S equations on unstructured grids. It relies on a third-order in space and time two-step Taylor-Galerkin scheme. A wall-adapting subgrid-scale viscosity based on the square of the velocity gradient tensor is used according to [26]. More details about the AVBP solver are available in $[35,16]$. The TurbFlow solver is primarily dedicated to turbomachinery flows and relies on a spatial discretization of the compressible NS equations based on finite volumes for multiblock structured grids. Convective fluxes are interpolated with a four-point centered scheme (fourth-order on regular grid) and diffusive fluxes with a two-point centered scheme (second order). Time marching relies on a five-step Runge-Kutta algorithm. The LES is handled by the SISM subgrid turbulence model as in our LB simulation. More details about the TurbFlow solver are available in [4].

The position of probes and measurement lines are identical in the experiment and simulations, as indicated in Fig. 6. Numerical samples are recorded during 180 vortex-shedding periods (after the transient regime) to ensure a satisfactory statistical convergence. The simulation is performed over $1.2 \times 10^{6}$ iterations on 256 processors and lasts about 39 hours including pre and post-processing tasks of the solver. The order of magnitude of this turnaround time is excellent as compared to turnaround times usually encountered with conventional CFD solvers. This results mainly from the computational simplicity of the LB scheme.

\section{Results of $\mathrm{LB}$ simulation}

In this section, our results are presented. First, a qualitative analysis of the flow is carried out. Then, more quantitative comparisons are made with experimental data and results obtained with $\mathrm{N}-\mathrm{S}$ computations on the same flow configuration. 


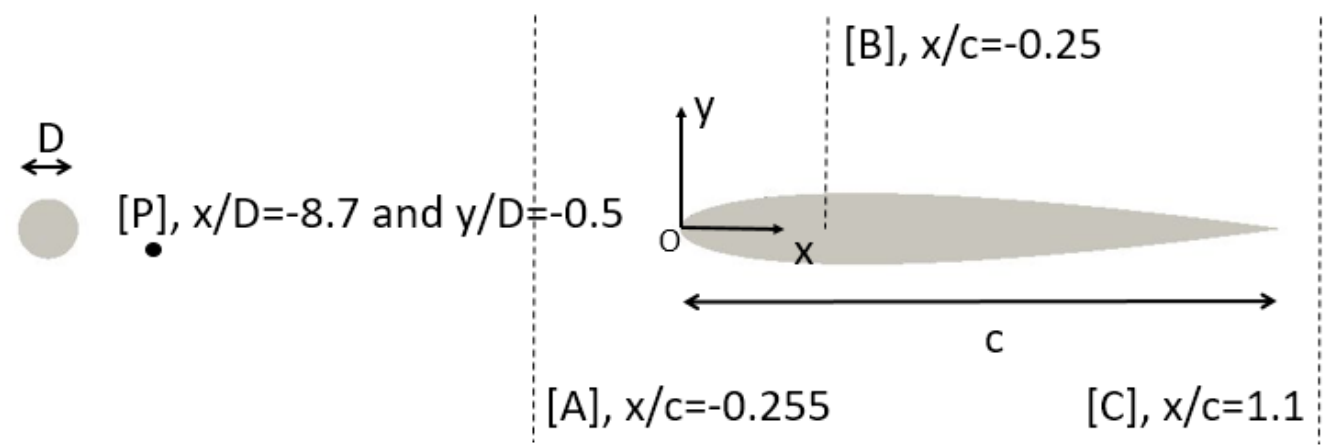

Figure 6: Velocity and pressure signals are recorded at position $\mathrm{P}$ for spectral analysis (in frequency). Velocity profiles are recorded along the lines A, B and C corresponding to the PIV measurements of the reference experiment.

\subsection{General view of the flow}

The complexity of the flow appears essentially in the interaction region between the rod and the airfoil. The contour of the time-averaged streamwise velocity indicates the presence of a recirculation bubble behind the rod, which contains a pair of counter-rotating vortices (see Figs. 7 and 8). These vortices do not interact directly with the airfoil. In the instantaneous flow, they alternatively detach from the rod, travel and eventually impinge on the leading edge of the airfoil (see Fig. 1). The length of the recirculation bubble given by $\mathrm{U}_{x}\left(\ell_{c}, 0\right)=0$ is well captured by our simulation (see Fig. 8). The rod is far enough from the airfoil and the near-wake is not perturbed by any blocking effect.

The overall structure of the turbulent flow is illustrated in Fig. 9 by an instantaneous snapshot of the spanwise velocity $\left(u_{z}\right)$. A turbulent von Kármán street originates from the transition of the shear layer that detaches from the rod. A broad range of turbulent scales is present in the wake. The vortex street eventually splits at the leading edge of the airfoil.

A nice property of the LB approach is to provide combined dynamic and acoustic fluctuations without resorting to any reconstruction or specific highlyaccurate numerical techniques, despite the large ratio between the length scales and levels of amplitude of turbulent fluid motion and acoustic-wave propagation. This property is made possible by the very low numerical dissipation of the LB scheme, in which integration is performed along characteristics. In Fig. 10, the radiated acoustic field, related to the aerodynamic field shown in Fig. 9, is displayed. Even if the computational domain does not allow for a propagation far 


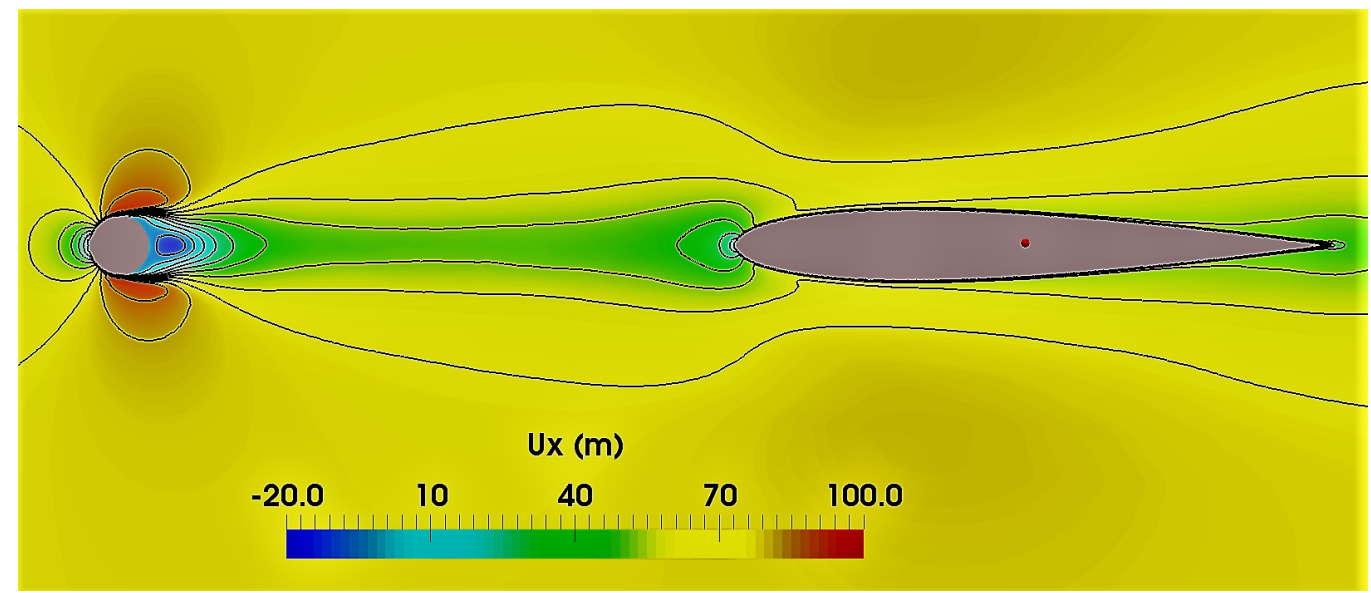

Figure 7: Contours of the time-averaged streamwise velocity $\left(u_{x}\right)$.

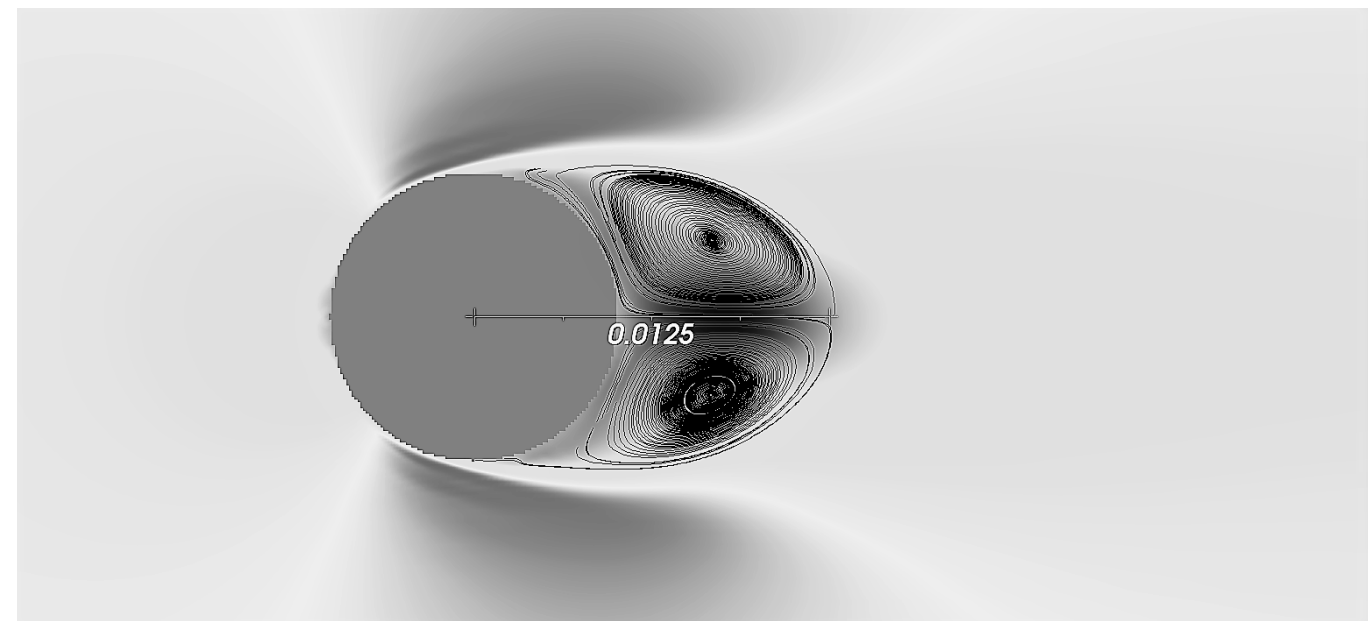

Figure 8: Mean-flow recirculation bubble in the near-wake of the rod (zooming view of Fig. 7). Velocity streamlines are displayed. The length of the bubble is in very good agreement with the reference value $\ell_{c} \approx 0.0125 \mathrm{~m}$ at $\operatorname{Re}_{\mathrm{D}}=48000$ [13].

from the rod-airfoil tandem, a tonal noise component associated with the periodic impingement of the von Kármán street on the airfoil is clearly observed. 


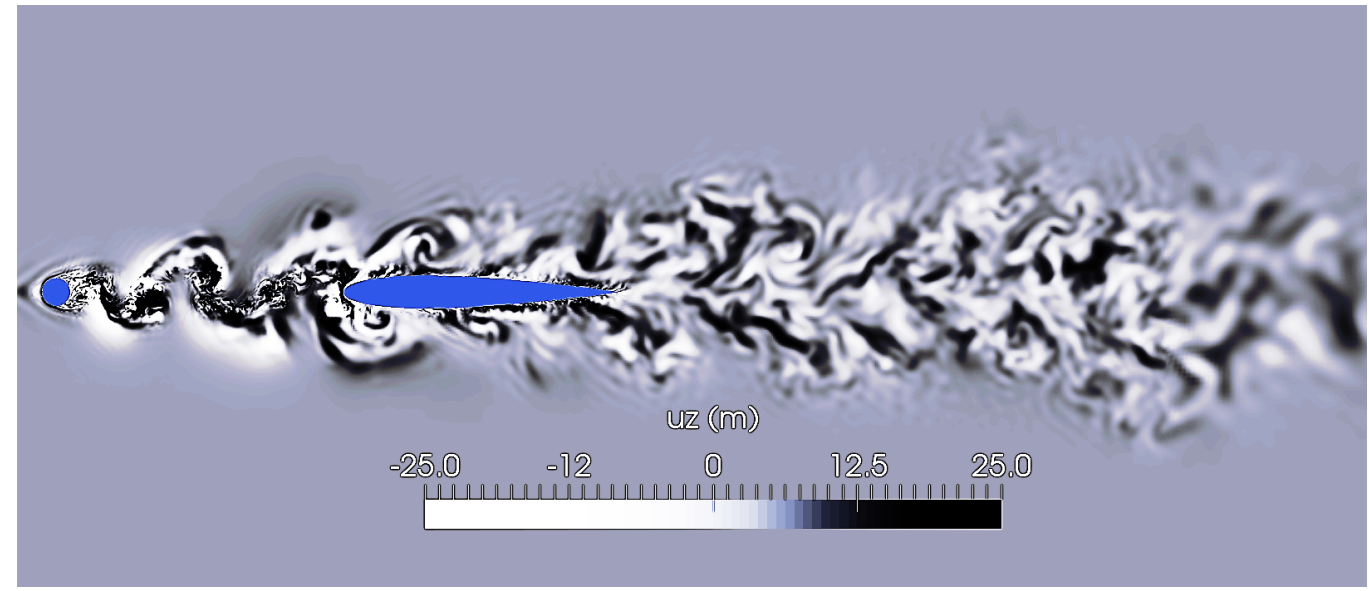

Figure 9: Instantaneous snapshot of the spanwise velocity $\left(u_{z}\right)$ in the central plane of the computational domain.

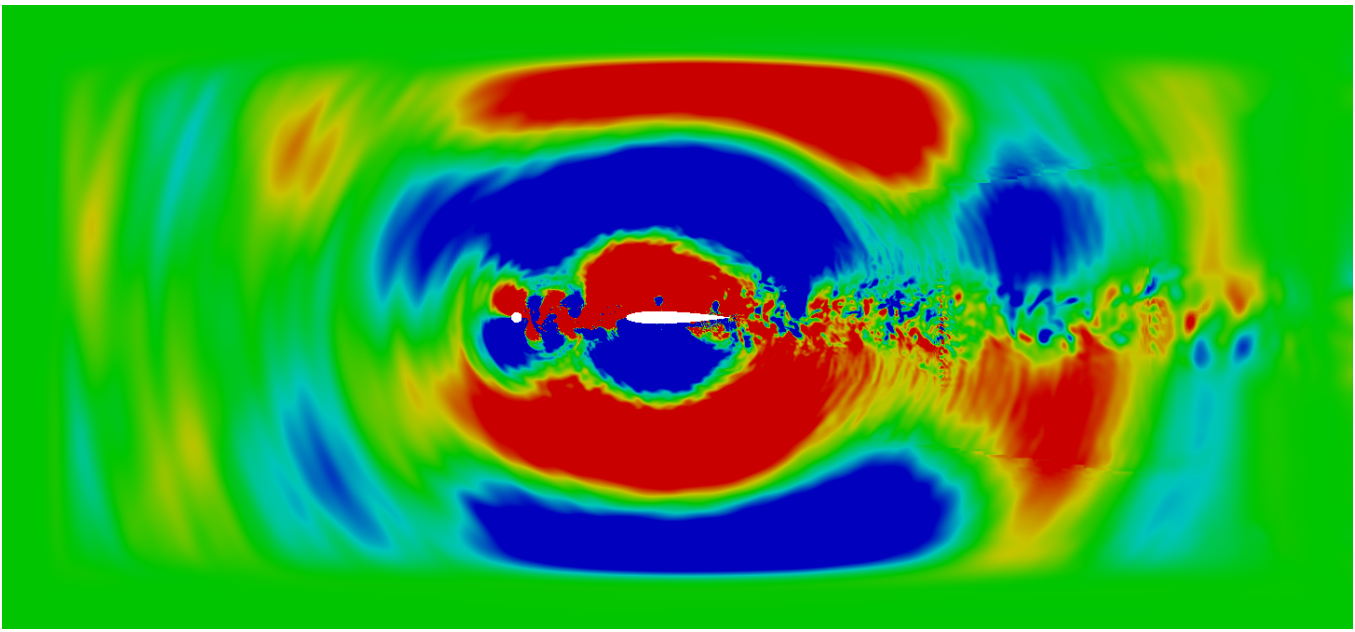

Figure 10: Instantaneous snapshot of the pressure fluctuation field $p^{\prime}(\mathbf{x}, t)=$ $p(\mathbf{x}, t)-\bar{p}(\mathbf{x})$ at the same instant as in Fig. 9. Colorscale from $-50 \mathrm{~Pa}$ (blue) to $+50 \mathrm{~Pa}$ (red). The whole computational domain is displayed. Dynamic absorbing layers (sponge zones) are used on the periphery of the domain [11].

\subsection{Vortex shedding and Strouhal number}

Since the flow is subject to both a quasi-periodic vortex shedding and broadband turbulence, a deeper insight is provided by a spectral analysis. The power 
spectral density of pressure fluctuations (as a function of the Strouhal number $\left.S t=f \cdot D / U_{\text {ref }}\right)$ in the interaction region between the rod and the airfoil is shown in Fig. 11. The spectrum includes both tonal and broadband components. The main tonal component appears at $S t=0.1915\left(f_{s}=1379 \mathrm{~Hz}\right)$ in agreement with the reference value reported in [14]. The expected wavelength of the radiated sound $\lambda_{s}=c_{s} / f_{s} \approx 2.5 c$ corresponds to our observation in Fig. 10.

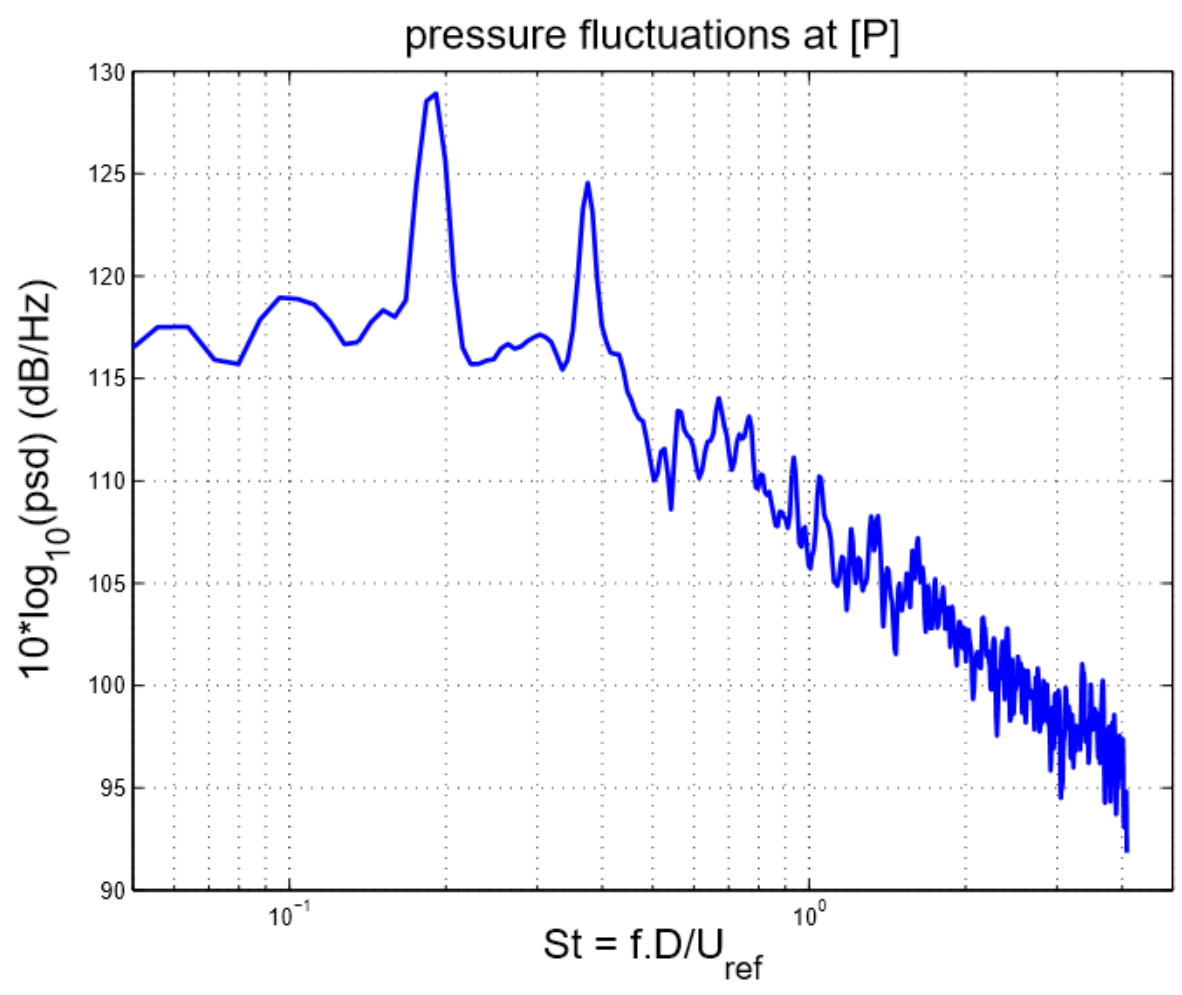

Figure 11: The power spectral density of the pressure fluctuations at position [P] in the turbulent von Kármán street between the rod and the airfoil (see Fig. 6).

The streamwise velocity spectrum at the same position is shown in Fig. 12. The fundamental vortex-shedding frequency is again clearly evidenced at $S t=$ 0.1915 . At higher frequencies, the spectral distribution of kinetic energy is close to the Kolmogorov's law $f^{-5 / 3}$ related to isotropic turbulence at high Reynolds number. The agreement with the hot-wire measurements carried out by Jacob et al. [18] is very satisfactory for the most energetic frequencies (see Fig. 12). 


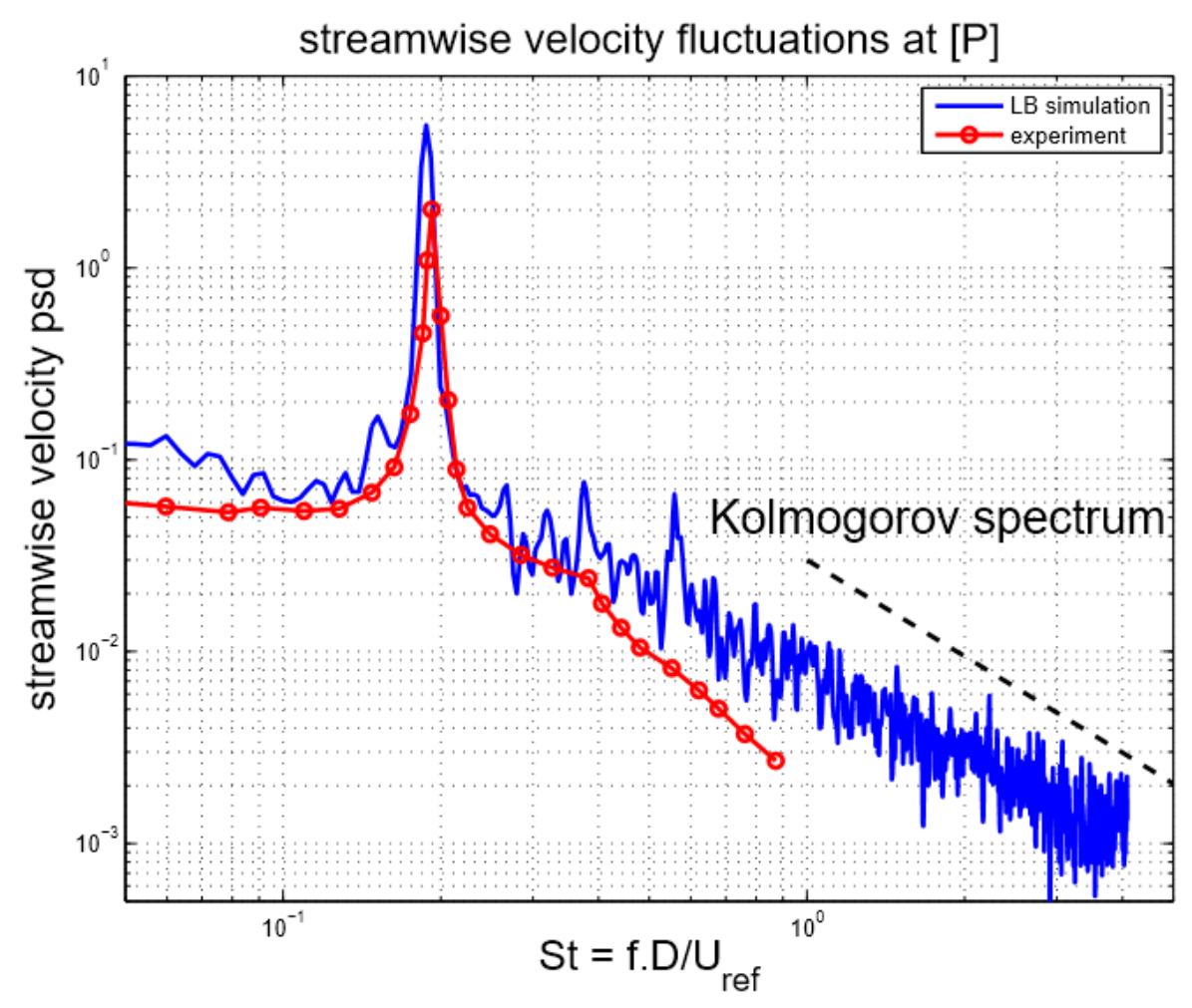

Figure 12: The power spectral density of the streamwise velocity at position [P]. LB simulation is compared with experimental data.

\subsection{Pressure on rod and airfoil}

The angular distributions of the mean pressure coefficient $C_{p}=\left(\bar{p}-p_{\infty}\right) / \frac{1}{2} \rho U_{\text {ref }}^{2}$ on the rod and on the airfoil are plotted in Figs. 13 and 14 respectively. Comparison is made with reference finite-volume Navier-Stokes LES (AVBP or TurbFlow). Note that our LB simulation (LaBS) relies on a wall-law model whereas NavierStokes LES is wall-resolved. The agreement is good for the rod and excellent for the airfoil.

The angular distributions of the root-mean-square pressure coefficient $C_{p}^{\prime}=$ $p_{\text {rms }} / \frac{1}{2} \rho U_{\text {ref }}^{2}$ are plotted in Figs. 15 and 16 . This quantity is more difficult to predict numerically. For the rod, the overall behavior is well-captured with a maximum close to the expected separation angle $\theta_{s} \approx 83^{\circ}$, as reported in the literature 


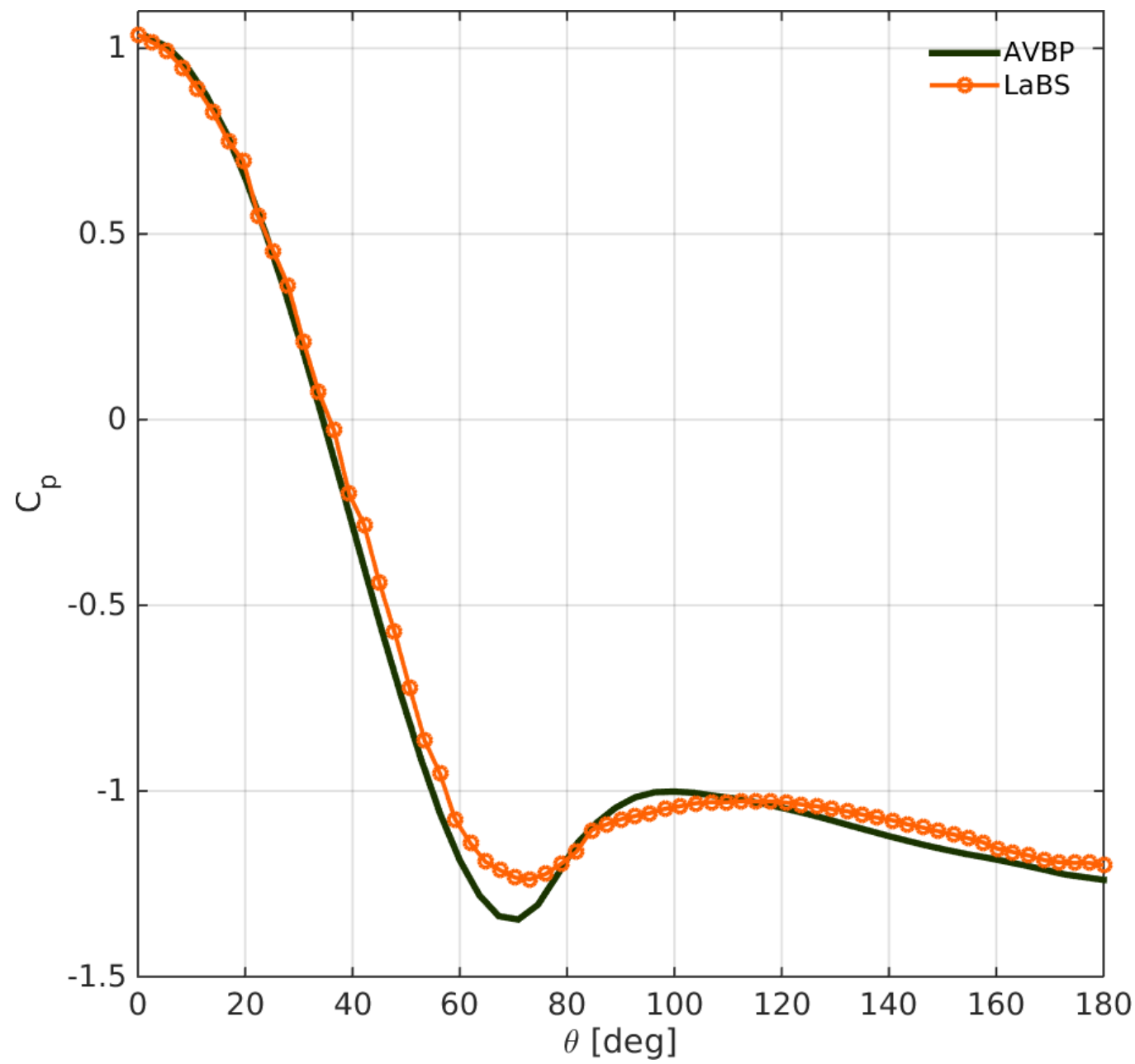

Figure 13: Mean pressure coefficient distribution on the rod. LB computation (LaBS) is compared with finite-volume simulation (AVBP).

for flows around circular cylinders in the range $4 \times 10^{4} \leq \operatorname{Re}_{\mathrm{D}} \leq 4.5 \times 10^{4}$ [43].

To better appreciate the observed discrepancy with the AVBP prediction, additional experimental and numerical results (flows around a circular cylinder at comparable Reynolds numbers) are shown in Fig. 15. Our result matches more closely with the TurbFlow prediction, which relies on the same subgrid-scale viscosity [5]. In general, we have a reasonable agreement with all the experimental and numerical predictions. The prediction of $C_{p}^{\prime}$ on the airfoil is much more satis- 


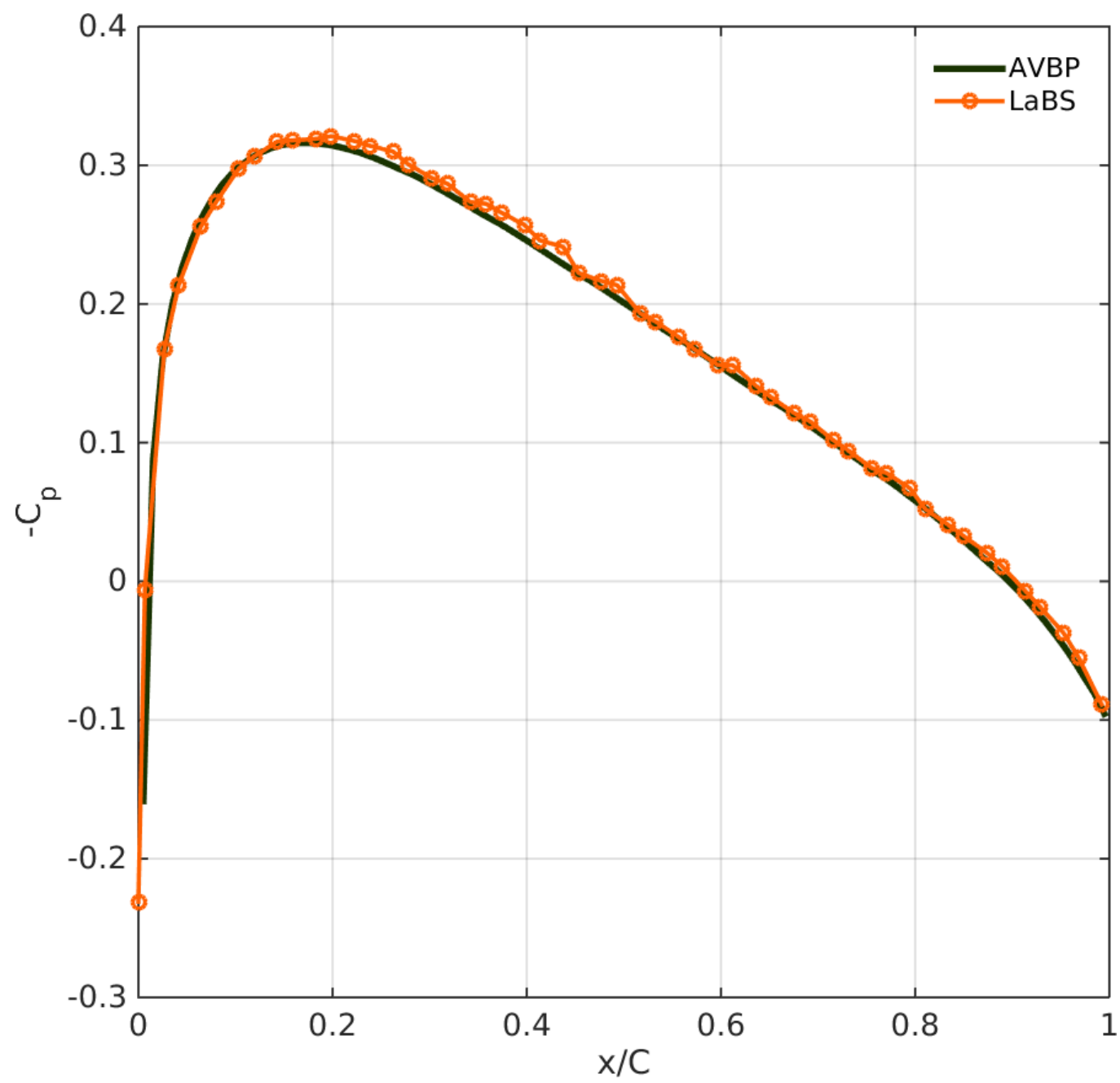

Figure 14: Mean pressure coefficient distribution on the airfoil. LB computation (LaBS) is compared with finite-volume simulation (AVBP).

factory (see Fig. 16). The amplitude of pressure fluctuations is slightly higher in the LB simulation, which may result from our wall-law modeling. 


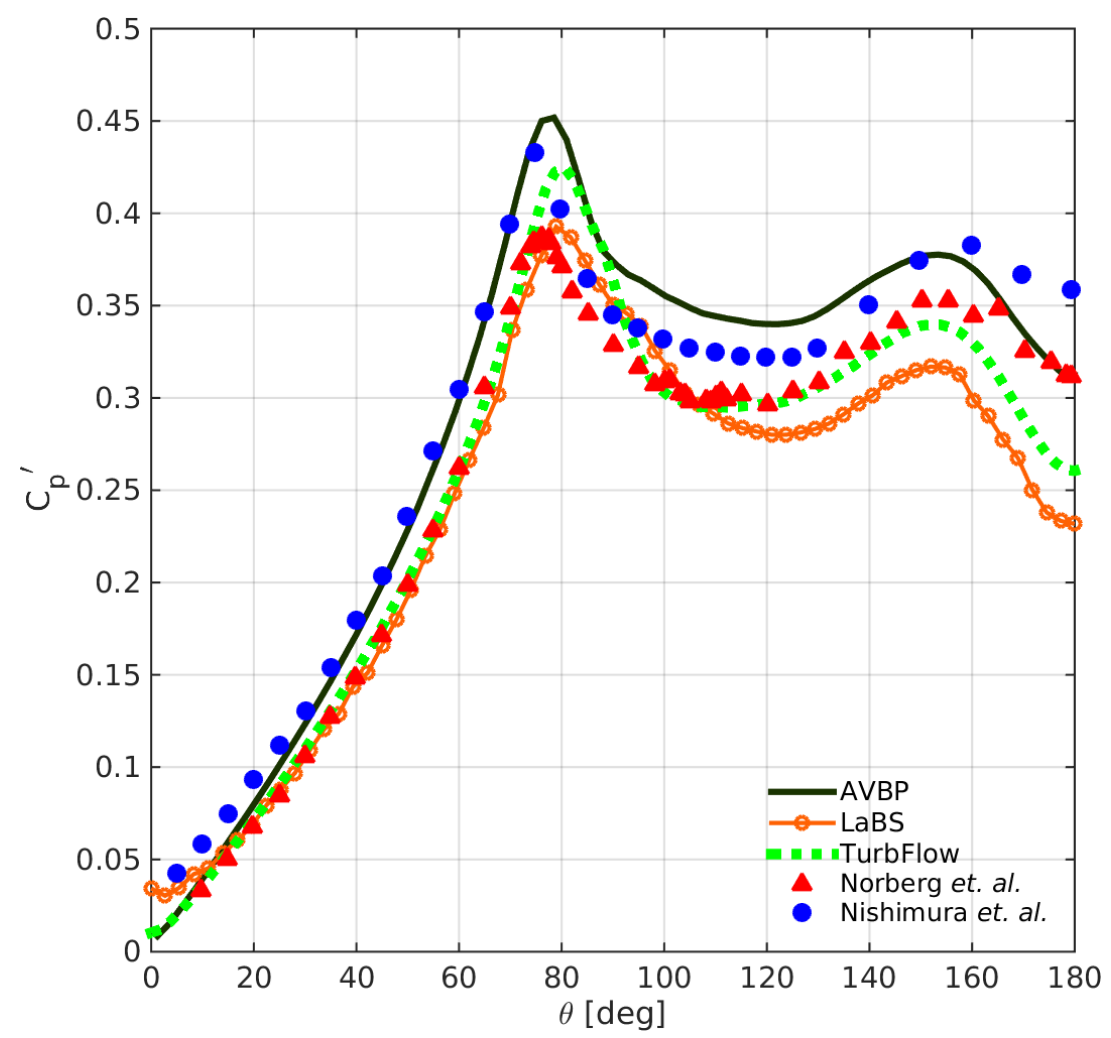

Figure 15: Root-mean-square pressure coefficient distribution on the rod. TurbFlow refers to a simulation at $\operatorname{Re}_{\mathrm{D}}=47000$ [5]; Norberg et al. [28] and Nishimura et al. [27] refer to experiments at $\mathrm{Re}_{\mathrm{D}}=61000$.

\subsection{Velocity and turbulence intensity}

The mean streamwise-velocity and turbulence intensity profiles, as a function of the normalized transverse coordinate $y / D$, are shown in Figs. 17 and 18 respectively. A satisfactory agreement is obtained with both the experimental and numerical reference data. Our results are closer to numerical data than experimental data. As already pointed out by Jacob et al. [18], a small error in the alignment of the rod-airfoil tandem (resulting in a $2^{\circ}$ angle of attack on the airfoil) may explain discrepancies with numerical predictions at positions [B] and [C].

Between the rod and the airfoil, the development of the wake is well captured with a correct turbulence intensity, slightly over-estimated compared to the experimental and other numerical data. Above the airfoil, the profiles are well- 


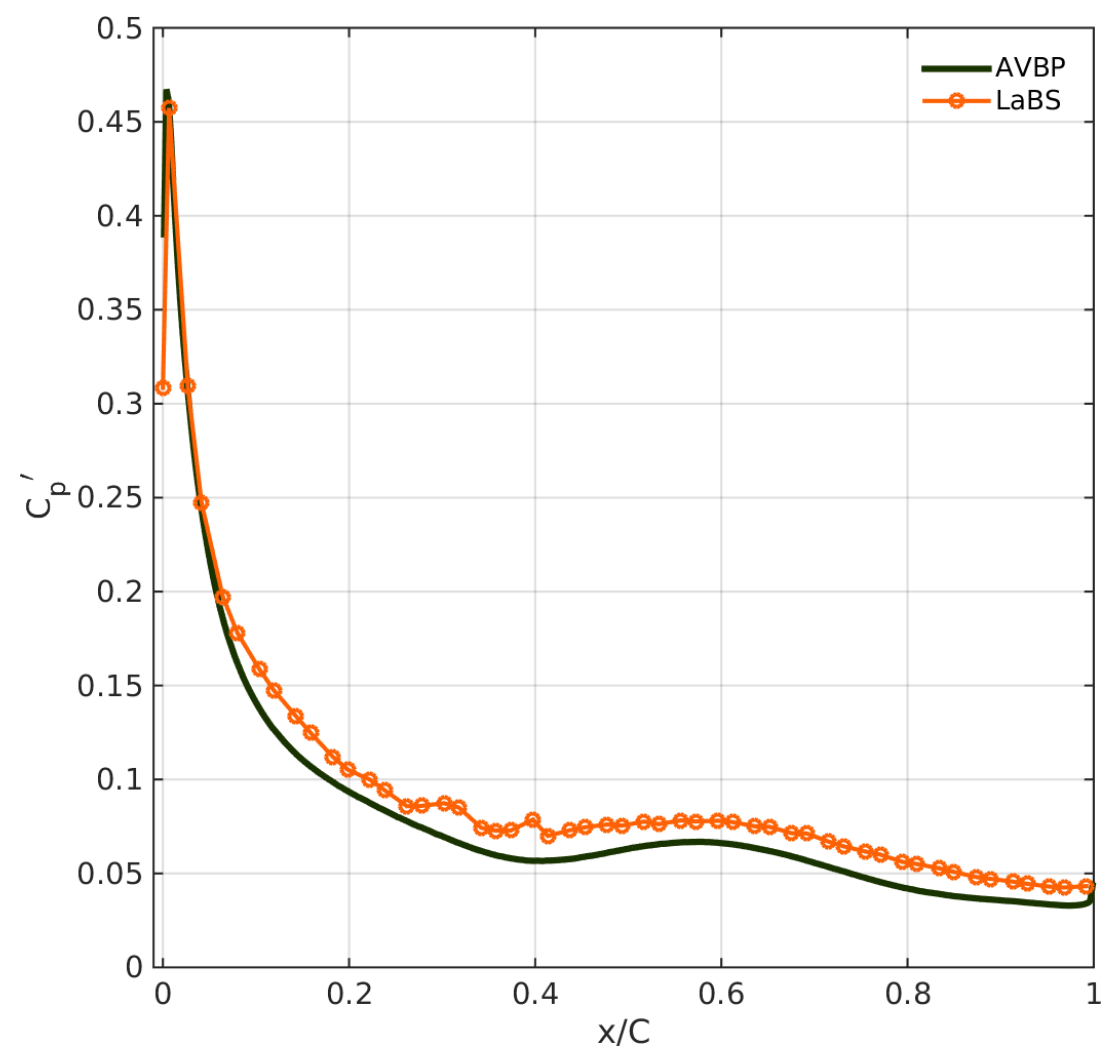

Figure 16: Root-mean-square pressure coefficient distribution along the airfoil. LB computation (LaBS) is compared with finite-volume simulation (AVBP).

reproduced despite the use of a wall-model in our LB simulation. Behind the airfoil, the results are also consistent with hot-wire measurements and AVBP predictions [16].

\subsection{Far-field acoustics}

Since small errors in the simulation of unsteady flows can result in large discrepancies in the far-field acoustic spectra, these are good indicators to assess the quality of the computation. The far-field pressure has been computed by using the Ffowcs-Williams and Hawkings (FWH) acoustic analogy [15, 8]. The integration here is performed on the rod and airfoil surfaces, thus neglecting the quadrupole sources (a priori justified at $\mathrm{Ma}_{\mathrm{ref}} \simeq 0.2$ ). The location of the observation probes 

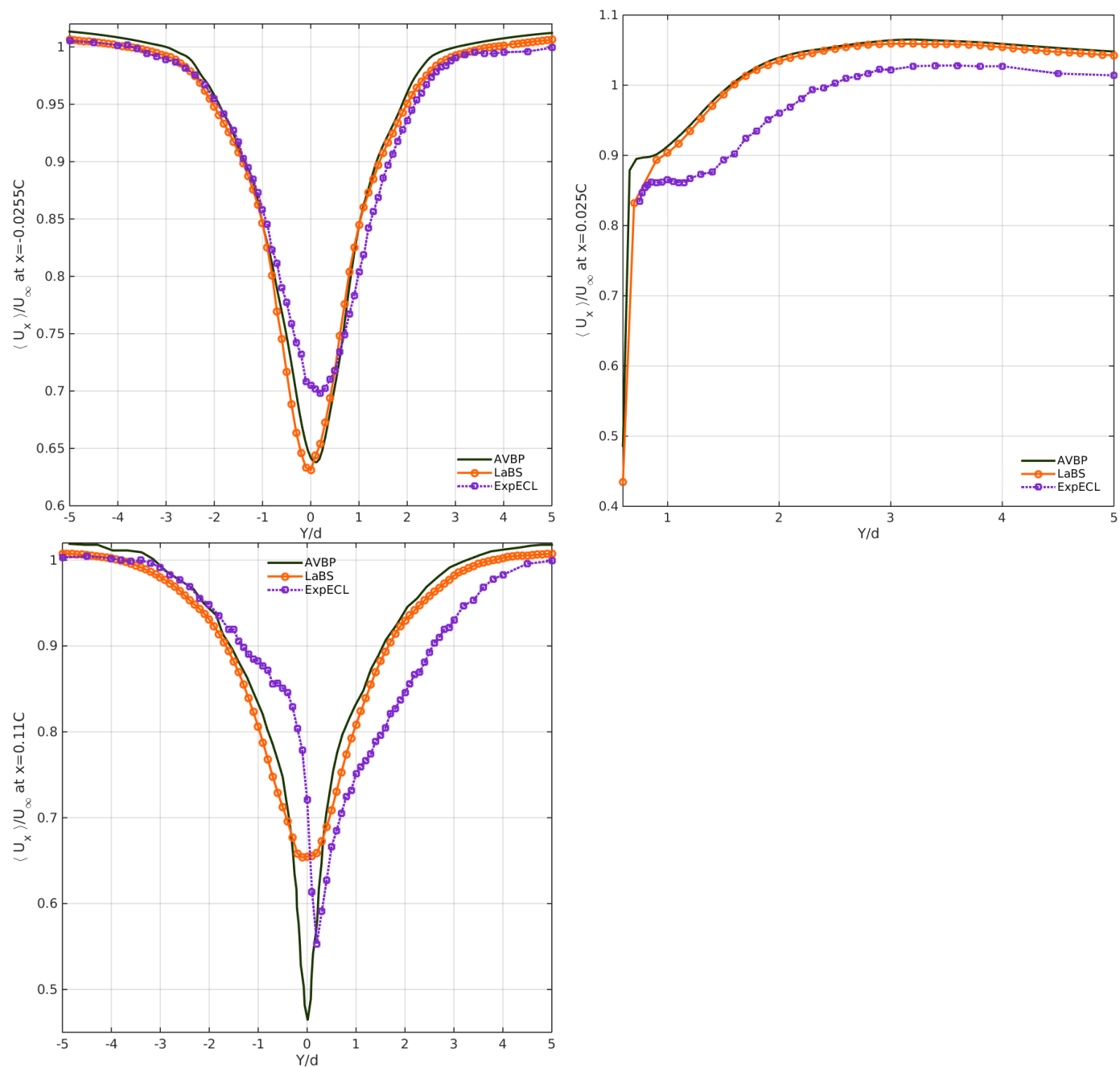

Figure 17: Mean velocity profiles along the lines $[\mathrm{A}],[\mathrm{B}]$ and $[\mathrm{C}]$. LB computation (LaBS) is compared with both finite-volume simulation (AVBP) and experimental data (ExpECL).

are the same as the ones used during the measurement campaign described in [18]. The FWH spectra are compared to the experimental data [18]. A correction is used to account for the span length of the rod-airfoil tandem, as explained in [20]. Fig. 19 presents the power spectral density of the far-field pressure fluctuations as a function of the Strouhal number $S t=f \cdot D / \mathrm{U}_{\text {ref }}$ for one of the probes located at position $x=c / 2$ and $y=18.5 c$. The general shape of the spectrum is 

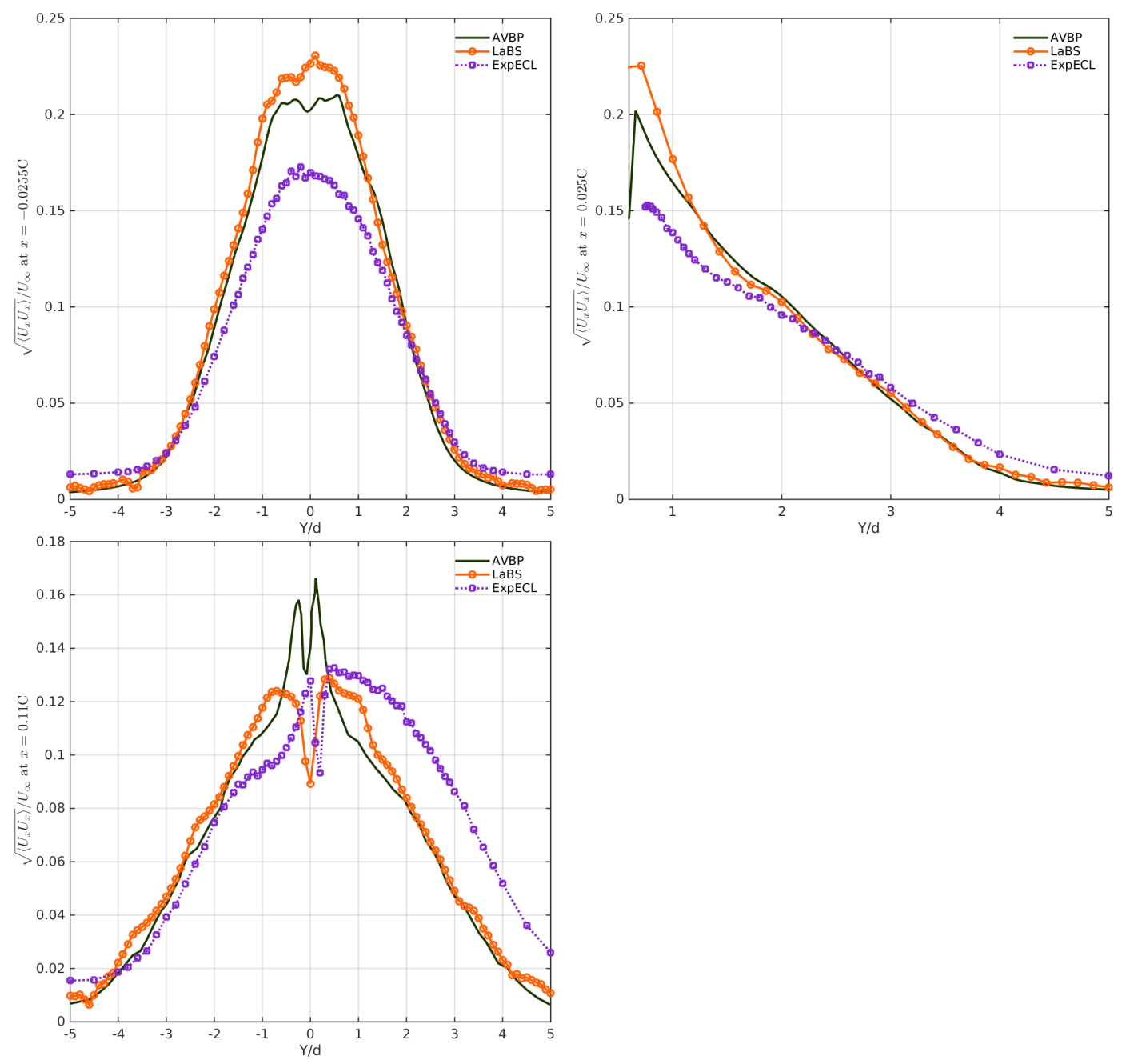

Figure 18: Turbulence intensity profiles along the lines $[\mathrm{A}],[\mathrm{B}]$ and $[\mathrm{C}]$. LB computation (LaBS) is compared with both finite-volume simulation (AVBP) and experimental data (ExpECL).

well reproduced by our simulation. It is dominated by a broad peak around the vortex-shedding frequency. The slope of the spectrum at high frequencies is captured. Overall, the agreement in the far-field is satisfactory for both fundamental peak and broadband components. The noise directivity has also been checked. The OverAll Sound Pressure Level (OASPL) is shown in Fig. 20 as a function of the angle at a radial distance $r=18.5 c$ from the airfoil center $x=c / 2$ and $y=0$. 
The difference between our computations and the experimental data is less than $3 \mathrm{~dB}$ for a broad range of angles, except for the range $90^{\circ}<\theta<110^{\circ}$ where the difference reaches $4 \sim 5 \mathrm{~dB}$. The computation and the experimental curves cross at $\theta=60^{\circ}$ and $\theta=120^{\circ}$. Below and beyond these angles, the experimental data decrease rapidly from $110 \mathrm{~dB}$ to $100 \mathrm{~dB}$ unlike the computational results. More work has to be done to understand this discrepancy, in particular the uncertainties that can have an effect on the directivity, e.g. a rod location bias.

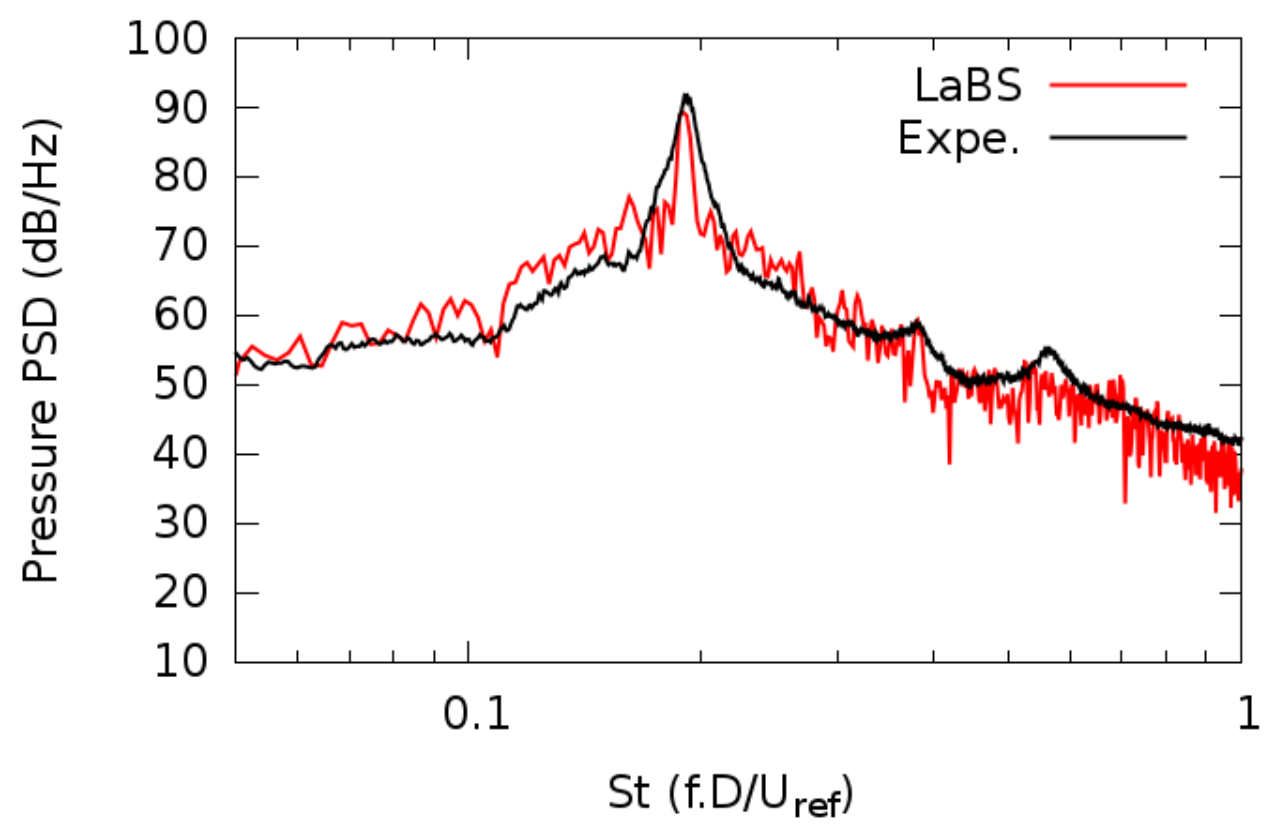

Figure 19: Power spectral density of pressure variations at the far-field position $x=c / 2$ and $y=18.5 c$. Pressure psd in $\mathrm{dB} / \mathrm{Hz}$ with reference pressure $2.10^{-5} \mathrm{~Pa}$.

\section{Conclusion}

This paper introduces an original CFD solver that incorporates a rich body of numerical methods and physical models, and has been used to simulate the flow past a rod-airfoil tandem in the sub-critical turbulent regime. The main difference with classical solvers is that it does not aim at solving the Navier-Stokes equations but a simplified discrete form of the Boltzmann equation. The collision operator 


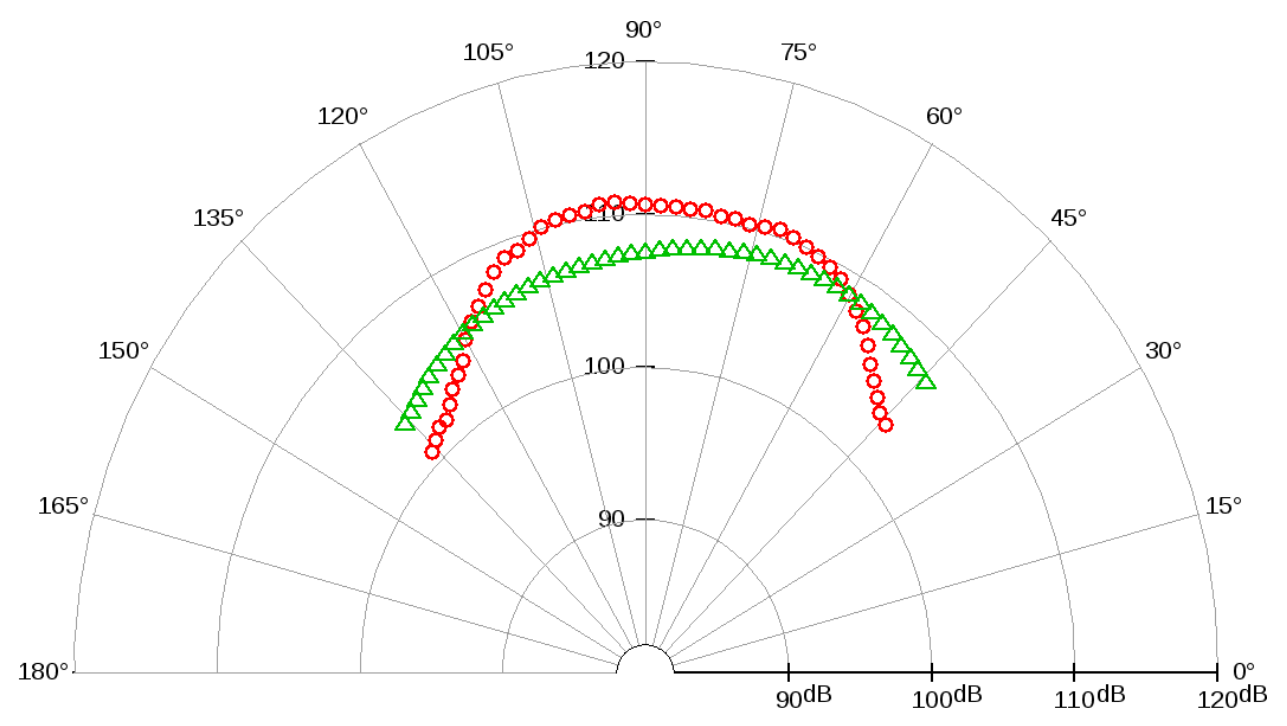

Figure 20: OASPL directivity around the airfoil center $(x=c / 2, y=0)$ at a radial distance $r=18.5 c$. Noise is expressed in $\mathrm{dB}$ versus the angle. The (red) circles refer to experimental data, whereas (green) triangles represent our numerical LB results.

here introduced is original and relies on two relaxation timescales for an improved stability at high Reynolds numbers; a second timescale is introduced to damp non-hydrodynamical modes. In order to tackle real-world industrial flows, two important physical ingredients have been included in the solver. First, an advanced subgrid turbulence model that suitably accounts for the unsteadiness and nonhomogeneity of the flow and allows us to obtain a reliable representation of largesized eddies and their dynamics. Second, an advanced wall-law model sensitive to the boundary curvature and pressure gradient that makes possible to dispense ourselves from the expensive integration of turbulent boundary layers.

The simulated flow compares fairly well on the basis of broad criteria with the original rod-airfoil experimental data of Jacob et al. and with alternative numerical results obtained with standard finite-volume N-S solvers. Unsteady pressure recordings on the rod and the airfoil has allowed us to propagate the acoustic noise generated by these bodies in the far field. The agreement is satisfactory between the reconstructed far-field acoustics and the experimental data, even if some small discrepancies still need to be elucidated.

This study underlines the attractiveness of the LB approach, which may actually be considered as a complementary tool to conventional CFD solvers. One 
may highlight the capability of the LB method to encompass dynamic and acoustic fluctuations in a simple numerical framework without resorting to any specific high-order numerical techniques. Therefore, when turnaround time is a concern, a LB solver becomes a serious contender because of its impressive computational efficiency. Finally, let us mention that the integration of more complex fluid physics is possible in the LB framework, which paves the path to efficient multi-physics CFD solvers [38].

\section{Acknowledgments}

LB simulations have been performed on the local HPC facilities at Ecole Centrale de Lyon (PMCS2I) and Ecole Normale Supérieure de Lyon (PSMN). These HPC facilities are supported by their academic host institutions, the Auvergne-RhôneAlpes region (GRANT CPRT07-13 CIRA) and the national Equip@ Meso grant (ANR-10-EQPX-29-01). The research work has been financially supported by the French Ministry of Industry and Bpifrance in the framework of the "Programme d'Investissement d'Avenir : Calcul Intensif et Simulation Numérique". F. Chevillotte and F.-X. Bécot (Matelys research Lab) have helped for the development of dynamic sponge zones in the LaBS solver. We thank Basile Bazin (CS-SI) for his help in the mesh setting.

\section{References}

[1] N. Afzal. Wake layer in a turbulent boundary layer with pressure gradient: A new approach. IUTAM Symposium on Asymptotic Methods for Turbulent Shear flows at High Reynolds Numbers, 535:95-118, 1996.

[2] C.K. Aidun and J.R. Clausen. Lattice-boltzmann method for complex flows. Annual Review of Fluid Mechanics, 42(439-472), 2010.

[3] P. L. Bhatnagar, E. P. Gross, and M. Krook. A Model for Collision Processes in Gases. I. Small Amplitude Processes in Charged and Neutral OneComponent Systems. Physical Review, 94:511-525, May 1954.

[4] J. Boudet, J. Caro, L. Shao, and E. Lévêque. Numerical studies towards practical large-eddy simulations. J. Therm. Sci., 16:328, 2007. 
[5] J. Boudet, E. Lévêque, P. Borgnat, A. Cahuzac, and M.C. Jacob. A kalman filter adapted to the estimation of mean gradients in the large-eddy simulation of unsteady turbulent flows. Comput. Fluids, 127:65-77, 2016.

[6] D.L. Brown and M.L. Minion. Performance Of Under-Resolved 2-Dimensional Incompressible-Flow Simulations. J. Comput. Phys., 122(1):165-183, NOV 1995.

[7] A. Cahuzac, J. Boudet, P. Borgnat, and E. Lévêque. Smoothing algorithms for mean-flow extraction in large-eddy simulation of complex turbulent flows. Phys. Fluids, 22:125104, 2010.

[8] D. Casalino. An advanced time approach for acoustic analogy predictions. Journal of Sound and Vibration, 261:583-612, 2003.

[9] H. Chen, S. Kandasamy, S. Orszag, R. Shock, S. Succi, and V. Yakhot. Extended boltzmann kinetic equation for turbulent flows. Science, 301:633-636, 2003.

[10] S. Chen and G.D. Doolen. Lattice Boltzmann method for fluid flows. Annual Review of Fluid Mechanics, 30(1):329-364, 1998.

[11] F. Chevillotte and D. Ricot. Development and evaluation of non-reflective boundary conditions for lattice boltzmann methods. 22nd AIAA/CEAS Aeroacoustics Conference, AIAA 2016-2915.

[12] P.J. Dellar. Bulk and shear viscosities in lattice Boltzmann equations. Phys. Rev. E, 64(3, 1):art. no.-031203, SEP 2001.

[13] H. Djeridi, M. Braza, R. Perrin, G. Harran, E. Cid, and S. Cazin. Near-wake turbulence properties around a circular cylinder at high reynolds number. Flow Turbul. Combust., 71:19, 2003.

[14] A. Eltaweel and M. Wang. Numerical simulation of broadband noise from airfoil-wake interaction. 17th AIAA/CEAS Aeroacoustics Conference, AIAA 2011-2802.

[15] J. E. Ffowcs-Williams and D. L. Hawkings. Sound generated by turbulence and surfaces in arbitrary motion. Philosophical Transactions of the Royal Society A, 264:321-342, 1969. 
[16] J. C. Giret, A. Sengissen, S. Moreau, M. Sanjosé, and J.-C. Jouhaud. Noise source analysis of a rod-airfoil configuration using unstructured large-eddy simulation. AIAA Journal, 53 (4):1062-1077, 2015.

[17] F. Higuera, S. Succi, and R. Benzi. Lattice gas-dynamics with enhanced collisions. Europhys. Lett., 9(4):345-349, 1989.

[18] M.C. Jacob, J. Boudet, J. Casalino, and M. Michard. A rod-airfoil experiment as benchmark for broadband noise modeling. J. Theoret. Comput. Fluid Dyn., 19 (3):171, 2005.

[19] Y. Jiang, M.-L. Mao, X.-G. Deng, and H.-Y Liu. Numerical investigation on body-wake flow interaction over rod-airfoil configuration. J. Fluid Mech., 779:1-35, 2015.

[20] C. Kato and M. Ikegawa. Large eddy simulation of unsteady turbulent wake of a circular cylinder using the finite element method. Advances in Numerical Simulation of Turbulent Flows, 1:49-56, 1991.

[21] J. Latt and B. Chopard. Lattice boltzmann method with regularized precollision distribution functions. Math. Comput. Simul., 72:165-168, 2006.

[22] E. Lévêque, F. Toschi, L. Shao, and J.-P. Bertoglio. Shear-improved smagorinsky model for large-eddy simulation of wall-bounded turbulent flows. J. Fluid Mech., 570:491-502, 2007.

[23] O. Malaspinas and P. Sagaut. Wall model for large-eddy simulation based on the lattice boltzmann method. J. Comput. Phys., 275:25-40, 2014.

[24] G.R. McNamara and G. Zanetti. Use of the boltzmann equation to simulate lattice-gas automata. Phys. Rev. Lett., 61:2332-2335, 1988.

[25] M.L. Minion and D.L. Brown. Performance of under-resolved twodimensional incompressible flow simulations, II. J. Comput. Phys., 138(2):734-765, DEC 1997.

[26] F. Nicoud and F. Ducros. Subgrid-scale stress modelling based on the square of the velocity gradient tensor. Flow Turbul. Combust., 62(3):183-200, 1999.

[27] H. Nishimura and Y. Taniike. Aerodynamic characteristics of fluctuating forces on a circular sylinder. J. Wind Eng Ind Aerodyn, 89:713-723, 2001. 
[28] C. Norberg. Fluctuating lift on a circular cylinder: review and new measurements. J. Fluids Struct, 17:57-96, 2003.

[29] V. C. Patel and F. Sotiropoulos. Longitudinal curvature effects in turbulent boundary layer. Prog. Aerospace Sci., 33 (1-2):1-70, 1997.

[30] D. Ricot, S. Marié, P. Sagaut, and C. Bailly. Lattice boltzmann method with selective viscosity filter. J. Comput. Phys., 228:4478-4490, 2009.

[31] P. Sagaut. Large eddy simulation for incompressible flows: An introduction. Springer-Verlag Berlin Heidelberg, 2006.

[32] P. Sagaut. Towards advanced subgrid models for lattice-boltzmann-based large-eddy simulation: theoretical formulation. Computers and Mathematics with Applications, 59(2194-2199), 2010.

[33] H. Sajjadia, M. Salmanzadeha, G. Ahmadib, and S. Jafaric. Simulations of indoor airflow and particle dispersion and deposition by the lattice boltzmann method using les and rans approaches. Build. Environ., 102:1-12, 2016.

[34] Rajani Satti, Phoi-Tack Lew, Yanbing Li, Richard Shock, and Swen Noelting. Unsteady flow computations and noise predictions on a rod-airfoil using lattice boltzmann method. 47th AIAA Aerospace Sciences Meeting Including The New Horizons Forum and Aerospace Exposition, AIAA 2009-497.

[35] T. Schönfeld and M. Rudgyard. Steady and unsteady flows simulations using the hybrid flow solver avbp. AIAA Journal, 37(11):1378-1385, 1999.

[36] Xiaowen Shan and Xiaoyi He. Discretization of the velocity space in the solution of the boltzmann equation. Phys. Rev. Lett., 80:65-68, 1998.

[37] S. Succi. The Lattice Boltzmann Equation for Fluid Dynamics and Beyond. Clarendon, 2001.

[38] S. Succi. Lattice boltzmann 2038. Euro. Phys. Lett., 109:50001, 2015.

[39] Michael Sukop and Daniel Thorne. Lattice Boltzmann Modeling. Springer, Berlin, Heidelberg, 2006. 
[40] H. Touil, D. Ricot, and E. Lévêque. Direct and large-eddy simulation of turbulent flows on composite multi-resolution grids by the lattice boltzmann method. J. Comput. Phys., 256:220-233, 2014.

[41] J. C. G. Verschaeve and B. Müller. A curved no-slip boundary condition for the lattice boltzmann method. J. Comput. Phys., 228:4478-4490, 2010.

[42] Z.J. Wang, Krzysztof Fidkowski, Remi Abgrall, Francesco Bassi, Doru Caraeni, Andrew Cary, Herman Deconinck, Ralf Hartmann, Koen Hillewaert, H.T. Huynh, Norbert Kroll, Georg May, Per-Olof Persson, Bram van Leer, and Miguel Visbal. High-order cfd methods: Current status and perspective. Int. J. Numer. Meth. Fluids, 00:1-42, 2012.

[43] M. M. Zdravkovich. Flow around circular cylinders. Oxford University Press, 2002. 